\title{
RNA Interference and MicroRNA Modulation for the Treatment of Cardiac Disorders
}

\section{Status and Challenges}

\author{
W. Poller(®), L. Suckau, S. Pinkert, and H. Fechner
}

\section{Contents}

1 Introduction to RNA-Based and Gene Therapeutic Strategies ...................................... 132

2 Key Issues of Current Vector Technologies ................................................................... 133

2.1 Targeting of Vectors for Regulatory RNAs or Genes .......................................... 134

2.2 Stability of Regulatory RNA or Transgene Expression ........................................... 136

2.3 Safety of RNA-Based and Gene Therapeutic Approaches ...................................... 136

3 RNA Interference Targeting Early Pathogenic Steps in Cardiomyopathies ..................... 137

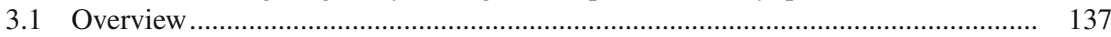

3.2 Introduction to Viral Cardiomyopathies ............................................................... 138

3.3 Results of an Anti-Virus Receptor Approach ....................................................... 139

3.4 Discussion of Anti-Viral Strategies .................................................................... 147

4 RNA Interference to Improve Cardiac Function in Advanced

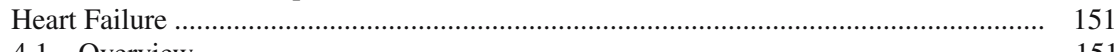

4.1 Overview .................................................................................................. 151

4.2 Regulatory RNA- and Gene-Based Therapies of Heart Failure.................................. 151

4.3 RNAi-Based Modulation of Cardiac $\mathrm{Ca}^{2+}$ Homeostasis
in Heart Failure ............................................................................................. 152

4.4 Discussion of an RNAi-Based Approach to Heart Failure Therapy ……................ 157

5 Clinical Perspectives and Unanswered Questions ........................................................ 159

5.1 Three Levels of Cardiac Targeting ........................................................................ 159

5.2 Stability and Control of Regulatory RNA and Gene Therapy ............................... 160

5.3 Practical Issues of Clinical Translation.................................................................... 160

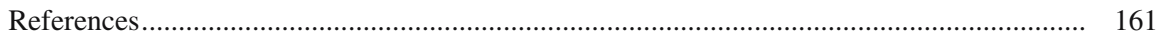

\begin{abstract}
The current status and challenges of RNA interference (RNAi) and microRNA modulation strategies for the treatment of myocardial disorders are discussed and related to the classical gene therapeutic approaches of the past decade. Section 2 summarizes the key issues of current vector technologies which determine if they may be suitable for clinical translation of experimental

\footnotetext{
W. Poller

Department of Cardiology and Pneumology, Campus-Benjamin Franklin, Charite University Medicine Berlin, Germany

e-mail: wolfgang.poller@charite.de
} 
RNAi or microRNA therapeutic protocols. We then present and discuss examples dealing with the potential of cardiac RNAi therapy. First, an approach to block a key early step in the pathogenesis of a virus-induced cardiomyopathy by RNAi targeting of a cellular receptor for cardiopathogenic viruses (Section 3). Second, an approach to improve cardiac function by RNAi targeting of late pathway of heart failure pathogenesis common to myocardial disorders of multiple etiologies. This strategy is directed at myocardial $\mathrm{Ca}^{2+}$ homeostasis which is disturbed in heart failure due to coronary heart disease, heart valve dysfunction, cardiac inflammation, or genetic defects (Section 4). Whereas the first type of strategies (directed at early pathogenesis) need to be tailor-made for each different type of pathomechanism, the second type (targeting late common pathways) has a much broader range of application. This advantage of the second type of approaches is of key importance since enormous efforts need to be undertaken before any regulatory RNA therapy enters the stage of possible clinical translation. If then the number of patients eligible for this protocol is large, the actual transformation of the experimental therapy into a new therapeutic option of clinical importance is far more likely to occur.

\begin{abstract}
Abbreviations AAV: Adeno-associated virus; AdV: Adenovirus; CAR: Coxsackievirus-adenovirus-receptor; DCM: Dilated cardiomyopathy; DCMi: Inflammatory cardiomyopathy; HF: Heart failure; miRNA: microRNA; misiRNA: miRNA-based shRNA; PLB: Phospholamban; RNAi: RNA interference; shRNA: Short hairpin RNA
\end{abstract}

\title{
1 Introduction to RNA-Based and Gene Therapeutic Strategies
}

In this chapter we will discuss the current status and challenges of using RNA interference (RNAi) and microRNA modulation strategies for the treatment of myocardial disorders and relate them to the classical gene therapeutic approaches of the past decade. The introductory Sect. 2 summarizes the key issues of current vector technologies which critically determine if they may be suitable for clinical translation of experimental RNAi or microRNA therapeutic protocols. We will then present and discuss two examples dealing with the potential of cardiac RNAi therapy. The first example deals with an attempt to block a very early step in the pathogenesis of a virus-induced cardiomyopathy and the resulting heart failure by RNAi targeting of a cellular receptor for cardiopathogenic viruses (Sect. 3). The second examples deals with an approach to improve cardiac function by RNAi targeting a late, common pathway of heart failure pathogenesis which is common to myocardial disorders of multiple etiologies. This strategy is directed at myocardiac $\mathrm{Ca}^{2+}$ homeostasis which is a major component of heart failure due to coronary heart disease (ischemic 
cardiomyopathy), heart valve dysfunction (valvular cardiomoypathy), cardiac inflammation (inflammatory cardiomyopathy), or genetic defects (dilated cardiomyopathy) (Sect. 4). Whereas the first type of strategies (directed at early pathogenic steps) needs to be tailor-made for each different type of pathogenesis, the second type (which target late common pathways) has a much broader range of application. This advantage of the second type of approach is of major importance since enormous efforts need to be undertaken before an RNAi or micro RNA-based therapeutic protocol enters the stage of possible clinical translation. If, then, the number of patients eligible for this protocol is large, the actual transformation of the experimental therapy into a new therapeutic option of major clinical importance is far more likely to occur.

\section{Key Issues of Current Vector Technologies}

The classical first concept of gene therapy which has been developed for monogenic disorders caused by deficiency of a single gene is gene substitution therapy. In the field of cardiology, several monogenic disorders primarily manifested in the myocardium could theoretically be cured by this approach. Among them are the various long-QT syndromes, the arrhythmogenic right ventricular dysplasia, the Brugada syndrome, the hypertrophic cardiomyopathies caused by a host of mutations in various sarcomeric proteins, and the dilated cardiomyopathies. A second concept of gene therapy is the enhancement of gene functions to cells, tissues, and organs by overexpression of primarily endogenous genes using gene transfer vectors. Under appropriate conditions, a therapeutic effect may be achieved not only in genetically determined but also in acquired diseases. A third concept is the addition of truly novel gene functions to the target organ by vectorbased expression of foreign genes. Novel concepts of far more recent origin involve the use of non-protein-coding, small regulatory RNA molecules for therapeutic purposes. They include the fourth concept of short hairpin RNA transcription from vectors for the suppression of gene functions in the diseased target organ or organism via the mechanism of RNA interference, and the fifth concept of using microRNA generation from vectors to achieve therapeutic modulation of cell and organ functions on the level of the cellular microRNA system (see Part I of this book). Naturally, all of the following key issues of classical gene therapy (concepts 1-3) also apply similarly to the RNA-based therapies (concepts 4 and 5). Figure 1 illustrates the fundamental difference in action between classical gene therapy vs. RNA therapy, but at the same time points to an important partial overlap between the two otherwise distinct strategies. Common to both is the need to deliver the therapeutic structure (cDNA-encoded protein vs. regulatory RNA) to the right place, at an appropriate concentration for a sufficient period of time, and with adequate safety features. This partial overlap allows to exploit advanced vector technologies, initially developed in the gene therapy field, for most types of RNA therapy, too. 


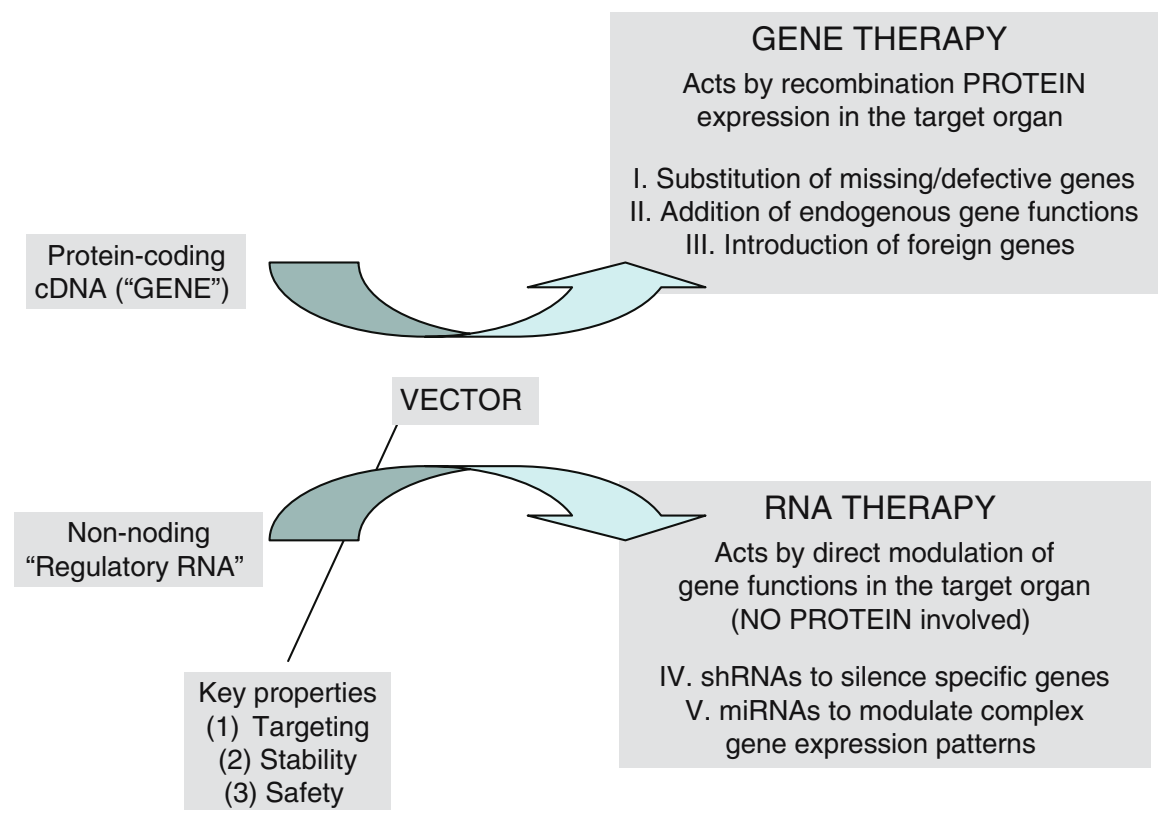

Fig. 1 Strategic Concepts of Gene Therapy versus Regulatory RNA Therapy

\subsection{Targeting of Vectors for Regulatory RNAs or Genes}

The first key issue of vector technology is the mere physical steering (targeting) of the vector (irrespective of expressing a protein-encoding cDNA, a short hairpin RNA or a pre-microRNA) to the target tissue, e.g., the myocardium, which proved to be a difficult task. Various approaches (Hoshijima et al. 2002; Rockman et al. 1998; White et al. 2000; Kypson et al. 1999; Koch et al. 2000; Ikeda et al. 2002) including intracoronary application (Giordano et al. 1996; Maurice et al. 1999) have been employed to achieve this goal, including already in clinical trials (YläHerttuala and Alitalo 2003; Simons et al. 2000; Grines et al. 2002; Hedman et al. 2003). A better understanding of the molecular and cellular determinants of vector targeting in the cardiovascular system has evolved during the past few years based on studies of the expression patterns of vector receptors (i.e. the receptors primarily mediating the cellular uptake of the virus from which the vector is derived) in animals (Fechner et al. 1999, 2003a) and humans (Noutsias et al. 2001; Poller et al. 2002a,b), and on the discovery of anatomical barriers such as the vascular endothelium or intracellular matrix (Fechner et al. 1999) inhibiting or preventing vector transfer to particular parts of the heart and other organs and tumors. Interestingly, the expression of a particularly important vector receptor, the Coxsackievirus- 
Adenovirus-Receptor (CAR), was found to be highly variable in human hearts (generalized induction in dilated cardiomyopathy) (Noutsias et al. 2001; Communal et al. 2003) and in animals (local induction after myocardial infarction) (Fechner et al. 2003a). Receptor-directed strategies (Curiel 1999) for the improvement of myocardial vector targeting have been evaluated but without significant success in vivo. If perfect physical vector targeting to the diseased tissue cannot be achieved, additional transcriptional confinement of the transgene may be achieved by using cardiac-specific promotors (Franz et al. 1997; Henderson et al. 1989; Reynolds et al. 2001). Recognition of the strong receptor-dependency of vector targeting has prompted investigation dealing with the possible therapeutic potential of tropism modifications by altering the receptor-interaction domains of the viruses used as vectors. A number of studies on the vector-susceptibility of different primary cell types for tropism modification have been conducted both for adenoviral (Wickham 2000; Wickham et al. 1997) and AAV vectors (Nicklin and Baker 2002; Rabinowitz et al. 2002; Ponnazhagan et al. 2002; Ponnazhagan and Hoover 2004; Shi and Bartlett 2003; Perabo et al. 2003; Büning et al. 2003). Basically, these approaches employ alterations of viral surface structures mediating cellular receptor binding and internalization of the vector. This has been achieved by genetic engineering of vector genes encoding vector surface components (Ogorelkova et al. 2006; Poller et al. 2002b), by bi-functional antibodies binding both to a vector surface epitope and a cellular structure specific for the desired target cells (Noutsias et al. 2001), or by bi-specific targeting proteins conjugated to the vector surface via avidin-biotin complexes (Perabo t al. 2003). However, none of these approaches has achieved similar success in vivo as the most recent generation of pseudotyped AAV vectors (in particular AAV8 and AAV9 for cardiac targeting), although use of these vectors may be combined with further tropism optimization by introducing additional alterations in the pseudotyped AAV capsids, and with tissue-specific promoters.

It should be emphasized that in order for the above tropism modifications to become useful in vivo the vectors first need to get direct access to the desired target cells, which may be prevented, however, by anatomical barriers (vascular endothelium, basal membrane, extracellular matrix). In addition to studies addressing the receptor issue, a number of approaches to overcome the anatomical barriers have been evaluated (Vale et al. 1999; Boekstegers et al. 2000; Beeri et al. 2002; Davidson et al. 2001; Price et al. 1998; Donahue et al. 1997; Hajjar et al. 1998), but none of these methods is simple or as yet appropriate for application in the clinical setting. In myocardial gene therapy, the final target cells - beyond the anatomical barriers - are particularly difficult targets as compared to other cell types and, at the current state of the art, viral vectors are the only option to achieve transfer rates sufficient for therapeutic efficacy. So far, no report on successful in vivo treatment of cardiac diseases using non-viral systems (including nanoparticles) has been published. Given the obstacles against efficient and targeted vector delivery, the recent development of imaging techniques for the in vivo detection of transgene expression may greatly facilitate the further development, monitoring, and assessment of cardiac gene therapeutic procedures (Wu et al. 2002; Inubushi et al. 2003; Auricchio et al. 2003). 


\subsection{Stability of Regulatory RNA or Transgene Expression}

The second key issue of vector technology is the stability of transgene function (be it transgenic protein expression or short hairpin or pre-microRNA transcription). If genetic therapy for heart failure is not only meant to serve as a bridge-totransplant or bridge-to-recovery, then long-term stability of the therapeutic vector plus transgene is required. Among the virus-based vector systems used for myocardial gene transfer, the currently most promising systems to achieve long-term stability are derived from adeno-associated viruses (AAVs). AAV vectors - although still difficult to produce at the high titers needed for in vivo applications - have shown stability for than a year in fully immunocompetent hemophilic dogs treated intramuscularly with a coagulation factor IX-producing AAV vector (Monahan et al. 1998; Herzog et al. 2002). Based on the experimental data a factor IX AAV vector has been evaluated in a clinical phase I trial (Kay et al. 2000). The high stability appears to be a consequence of inherent fundamental biological properties of the AAV genome (Afione et al. 1996). Although it is not always clear whether transgene expression occurs from integrated or episomal vector genomes, sequencing of vector-genome junctions has demonstrated the presence of integrated AAV genomes in cultured human cells and in mice after treatment with AAV vectors (Miller et al. 2002; Rutledge and Russell 1997; Nakai et al. 1999, 2001, 2003). AAV vectors have only recently been employed for the cardiac genetic treatment of heart failure (Hoshijima et al. 2002), for the systemic treatment of Fabry disease (Takahashi et al. 2002), and for substitution gene therapy of an inherited cardiomyopathy due to a $\delta$-sarcoglycan gene defect (Kawada et al. 2002), with transgene expression over several months in all cases.

\subsection{Safety of RNA-Based and Gene Therapeutic Approaches}

The third key of vector technology - of paramount importance before any clinical application may be considered - is the safety of the gene transfer protocols. One safety feature under intense investigation is approaches towards regulation of the transgene after injection into the host. This may not be required for any application but is necessary in situations when adaptation of transgene activity to the physiologically required level is desired. An option to shut down the transgene completely at any time in the case of serious adverse effects is highly desirable under safety considerations (Bliznakov 2002). Vectors systems that can be shut off by withdrawal of an inducer drug have been described, most of them using doxycycline as inducer (Fechner et al. 2003b; Srour et al. 2003; Chtarto et al. 2003).

Safety issues inherent to specific vector types are, e.g., the inflammatory responses against adenoviral vectors which were transient, however, and appeared to be of no clinical significance in two recent cardiological clinical trials in 2002 and 2003 employing intracoronary injection of adenoviral vectors expressing VEGF (Grines et al. 2002; Hedman et al. 2003). In a previous trial in the year 1999 
(Raper et al. 2002); a liver-targeted gene therapy protocol aiming at the treatment of ornithin transcarboxylase deficiency had used vector doses three orders of magnitude higher than those in the recent cardiological trials. The highest dose used therein was $3.3 \times 1,010$ vector particles (Grines et al. 2002), whereas in the former liver-directed study the multiorgan failure and death occurred in one patient who had received the highest dose of $3.8 \times 1,013$ particles (Raper et al. 2002). That tragic event prompted thorough additional investigations (Lehrman 1999; Marshall 2000) into the risks of adenovector-mediated gene therapy including serious adverse events even if they may occur only very rarely, in predisposed individuals, or at very high vector doses. A survey of clinical trials in 100 cancer patients employing intravascular adenoviral vectors reports that doses up to $2.5 \times 1,013 \mathrm{had}$ an acceptable safety profile (Reid et al. 2002). Even very rare serious side effects will be considered unacceptable, however, for non-malignant diseases.

The safety of AAV vector-based cardiac gene therapy is currently under investigation in a Phase I safety trial at the Mount Sinai Hospital in New York (unpublished data). With respect to possible risks specific to the AAV vectors their immunogenicity is low as compared to adenovectors (Sun et al. 2002, 2003). Another possible risk has been deduced from the capacity of wild-type AAV for chromosomal integration which appears to be lost, however, in recombinant AAV vectors such as used for gene therapy (Rutledge and Russell 1997; Rutledge et al. 1998; Miller et al. 2002, 2004). A recent study has searched for possible chromosomal effects of AAV vector integration (Miller et al. 2002) which deserve particular attention after a recent report on the late and unexpected occurrence of leukemia in children treated with retroviral vectors for severe combined immmunodeficiency (SCID), after successful correction of the primary genetic defect (Kohn et al. 2003). Whereas retroviral vectors such as used in the SCID study are integrating into the human genome at random sites, wild-type AAV shows a preference for chromosome 19 (Miller et al. 2002; Nakai et al. 1999). The possible consequences of genomic integration of AAV vectors deserves close attention over years, since the retrovector-associated leukemia occurred only years after successful ex vivo gene therapy for SCID by retrovirally mediated transfer of the $\gamma \mathrm{c}$ gene into CD34+ cells (Kohn et al. 2003; Check 2003; Hacein-Bey-Abina et al. 2002, 2003).

\section{RNA Interference Targeting Early Pathogenic Steps in Cardiomyopathies}

\subsection{Overview}

Since coxsackievirus B3 (CoxB3) and adenoviruses may cause acute myocarditis and inflammatory cardiomyopathy, isolation of the common CoxsackievirusAdenovirus-Receptor (CAR) has provided an interesting new target for molecular antiviral therapy. Whereas many viruses show high mutation rates enabling them to 
develop escape mutants, mutations of their cellular virus receptors are far less likely. We report on antiviral efficacies of CAR gene silencing by short hairpin (sh)RNAs in the cardiac-derived HL-1 cell line and in primary neonatal rat cardiomyocytes (PNCMs). Treatment with CAR-shRNA-generating vectors resulted in almost complete silencing of CAR expression both in HL-1 cells and PNCMs. While in HL-1 cells, CAR was already silenced $24 \mathrm{~h}$ after transduction of CARshRNA expressing vector in PNCMs CAR downregulation becomes visible only at day 6 and thereafter. CAR knockout resulted in strong inhibition of CoxB3 infections by up to $97 \%$ in HL-1 cells and by up to $90 \%$ in PNCMs, while adenovirus infections were inhibited by only $75 \%$ in HL-1 cells but up to $92 \%$ in PNCMs. We conclude that CAR knockout by shRNA vectors is promising against CoxB3 and adenovirus infections, but cell type specific CAR silencing by vector expressed shRNAs needs to be considered for the antiviral approaches.

\subsection{Introduction to Viral Cardiomyopathies}

Initially, cardiac viral infections were documented in the clinical context of acute myocarditis. Coxsackievirus B3 (CoxB3) was the first virus detected in this condition in humans (Bowles et al. 1986), and adenoviruses of serotypes 2 and 5 were later described as common agents of myocarditis in children (Bowles et al. 2003). Systematic screening of patients with dilated cardiomyopathy (DCM) has recently revealed that a rather broad spectrum of viruses may also chronically persist in human myocardium (Kühl et al. 2005a). It is therefore assumed that acute cardiac viral infections may not only cause acute illness, but also chronic heart disease if not definitely eliminated from the heart (Kühl et al. 2005b). The disease may progress to terminal heart failure and then require heart transplantation as a last therapeutic option.

RNA interference (RNAi) is a process of posttranscriptional gene silencing mediated by double-stranded RNA (dsRNA). The introduction of double-stranded small interfering RNAs (siRNA) or vectors expressing short hairpin RNA (shRNA) have already been successfully used to inhibit the replication of multiple viruses in vitro and in vivo including respiratory viruses (Bitko et al. 2005), hepatitis B (Carmona et al. 2006; Weinberg et al. 2007; Chen et al. 2007) and C viruses (Takigawa et al. 2004), human herpes virus-6 (Yoon et al. 2004), cytomegalovirus (Wiebusch et al. 2004), SARS coronarvirus (Lu et al. 2004), HIV-1 (Hayafune et al. 2006a,b) and CoxB3 (Merl et al. 2005; Werk et al. 2005). However, the efficiency of RNAi depends on exact homology of the siRNA to the target sequence. A single mismatch can be sufficient to abrogate silencing by RNAi (Sabariegos et al. 2006).

Many RNA viruses encode polymerase enzymes that lack proof reading abilities and as a result have a high mutations rate. Thus, there is a high probability that viruses will rapidly develop resistance to a particular siRNA during virus replication by incorporation of nucleotide mutations within the target sequence of the siRNA. Furthermore, it has been shown that hepatitis $\mathrm{C}$ virus becomes resistant 
against a particular siRNA after several cycles of replication by the incorporation of point mutations within the siRNA target sequence (Wilson and Richardson 2005). Similar data have been reported for HIV-1 (Boden et al. 2003, 2007) and poliovirus infections (Gitlin et al. 2005). Therefore, therapeutic targeting of nonvariable virus-binding receptors on susceptible cells or other host cell molecules directly or indirectly involved in virus infections is an interesting alternative to targeting of the virus itself (Werk et al. 2005; Murray et al. 2005; Anderson and Akkina 2005; Arrighi et al. 2004; Gao et al. 2004; Ping et al. 2004; Kameoka et al. 2004). One approach of this type showed that downregulation of the CD4-independent attachment receptor (DC-SIGN) significantly inhibited HIV infection of dendritic cells (DC) and prevented the transfer of infectious HIV-1 particles from DC to T cells (Arrighi et al. 2004).

Adenoviruses of types 2 and 5 and CoxB3 use the Coxsackievirus-AdenovirusReceptor (CAR) to infect their target cells, either for virus attachment (adenoviruses) or virus internalization (CoxB3) (Bergelson et al. 1998). Therefore, CAR represents an attractive target for the inhibition of cardiac CoxB3 and adenovirus infections. It has been shown recently that suppression of CAR by RNAi results in inhibition of CoxB3 infections (Werk et al. 2005; Coyne and Bergelson 2006). These studies were carried out with cells of non-cardiac origin and chemically synthesized siRNAs were used to inhibit CAR expression. However, the therapeutic use of synthetic siRNAs is significantly limited by their rapid degradation in target cells resulting in only transient gene silencing (Watanabe et al. 2004). Moreover, most cells of cardiac origin are very difficult to transfect with chemically synthesized siRNAs even in vitro. These problems are significantly aggravated in vivo. Despite intense efforts during recent years systemic and target organ-specific delivery of siRNAs remains a major hurdle for in vivo applications of RNAi.

Therefore, we investigated the antiviral potential of CAR gene silencing in cells of cardiac origin by use of newly developed AdVs generating shRNA against murine or rat CAR (shCAR). shCAR treatment of HL-1 cells and primary neonatal rat cardiomyocytes (PNCMs) led to almost complete silencing of CAR gene expression and efficient inhibition of CoxB3 infection, while efficient inhibition of adenovirus infections was only seen in PNCMs. The studies also revealed cell type-specific responses of cellular CAR protein to shCAR treatment which need to be considered as important determinants of this new antiviral approach in vivo.

\subsection{Results of an Anti-Virus Receptor Approach}

\subsubsection{Selection and Specificity of Mouse CAR shRNAs}

To investigate the efficacy of CAR silencing for the inhibition of adenovirus and CoxB3 infection in cardiac HL-1 cells, we generated a total of three shRNAs (shCAR2m, shCAR3m, shCAR4m) directed against extracellular domains of the 


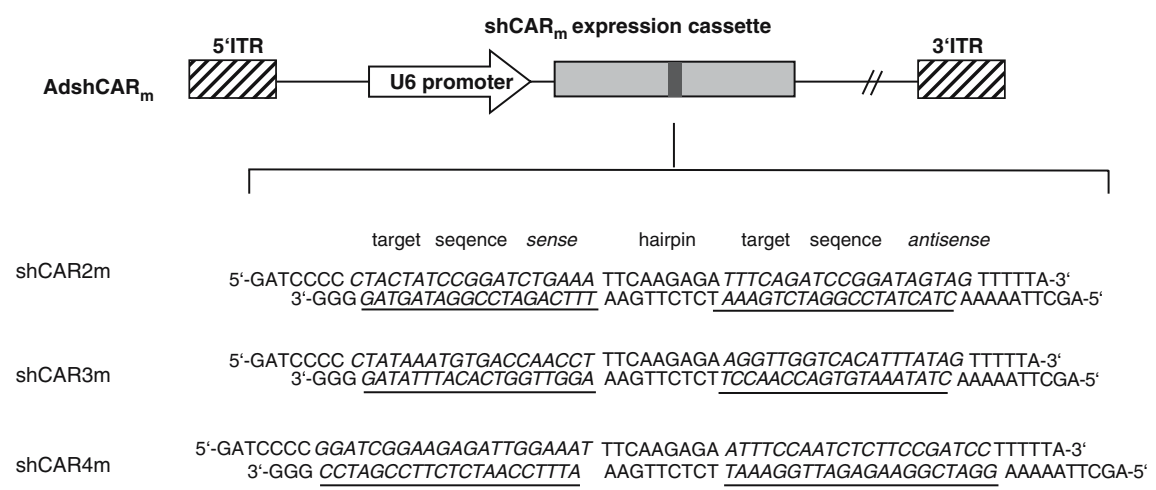

Fig. 2 Structure of adenovectors for shRNA transcription. Schematic illustration of recombinant AdshCARm and the sequence of shCAR: the 5'-ITR represents nucleotide positions 1-342 of adenovirus type 5; the shCAR expression cassette is composed of an U6 = RNA polymerase III promoter and an shCAR sequence; the individual shCAR motifs consist of 19 (shCAR2m, $s h C A R 3 m)$ or 21 nucleotides $(s h C A R 4 m)$ corresponding to the coding regions of mCAR1 and mCAR2. The two motifs that form the sense and antisense strand of the shRNA are separated by a loop of nine nucleotides. A transcriptional termination signal of five tymidines is added at the $3^{\prime}$-end of the inverted repeat. Whereas shCAR2 $m$ and shCAR $3 m$ are directed against the IG1 extra-

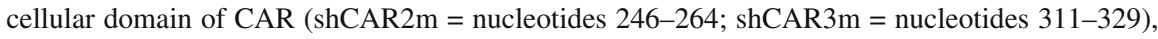
shCAR4m is directed against the nucleotides $448-468$ of the IG2 extracellular domain; $3^{\prime}$-ITR right inverted terminal repeat of adenovirus 5. Note: siRNA sequence in shCAR2m matches complete to mouse and rat CAR target sequences

mCAR splice variants mCAR-1 (Bergelson et al. 1998) and mCAR-2 (Tomko et al. 1997) (Fig. 2) following published siRNA selection criteria (Reynolds et al. 2004). DNA oligonucleotides encoding these shRNAs were then cloned into expression plasmids under the control of a murine RNA polymerase III U6 promotor. To test their efficacies, we co-transfected 293T cells with shCAR2m, shCAR3m, or shCAR4m-expressing plasmid, together with plasmids expressing recombinant mCAR1 and mCAR2. As a transfection control a plasmid expressing an irrelevant shRNA was used. To determine the extent of recombinant mCAR1- and mCAR2mRNA downregulation, total cellular RNA was isolated $48 \mathrm{~h}$ after transfection and Northern blot hybridization was carried out. These experiments showed that two shRNAs were highly efficient: shCAR2m with $93 \%$ silencing, and shCAR4m with 97\% silencing of mCAR1- and mCAR2-mRNA. The control shRNA affected neither mCAR1- nor mCAR2-mRNA expression (Fig. 3a). To analyze the specificities of shCAR2m and shCAR4m, both were also tested for downregulation of human CAR (hCAR) by co-transfection with a plasmid expressing recombinant soluble hCAR. None of the mouse CAR-specific shRNAs influenced human CAR-mRNA expression. In contrast, a human-specific shRNA efficiently silenced hCAR-mRNA expression (Fig. 3b). Considering that shCAR $2 \mathrm{~m}$ has only one mismatch and shCAR4m three mismatches as compared to hCAR-mRNA, these experiments indicate very high species-specificity of the selected mCAR-shRNAs. 

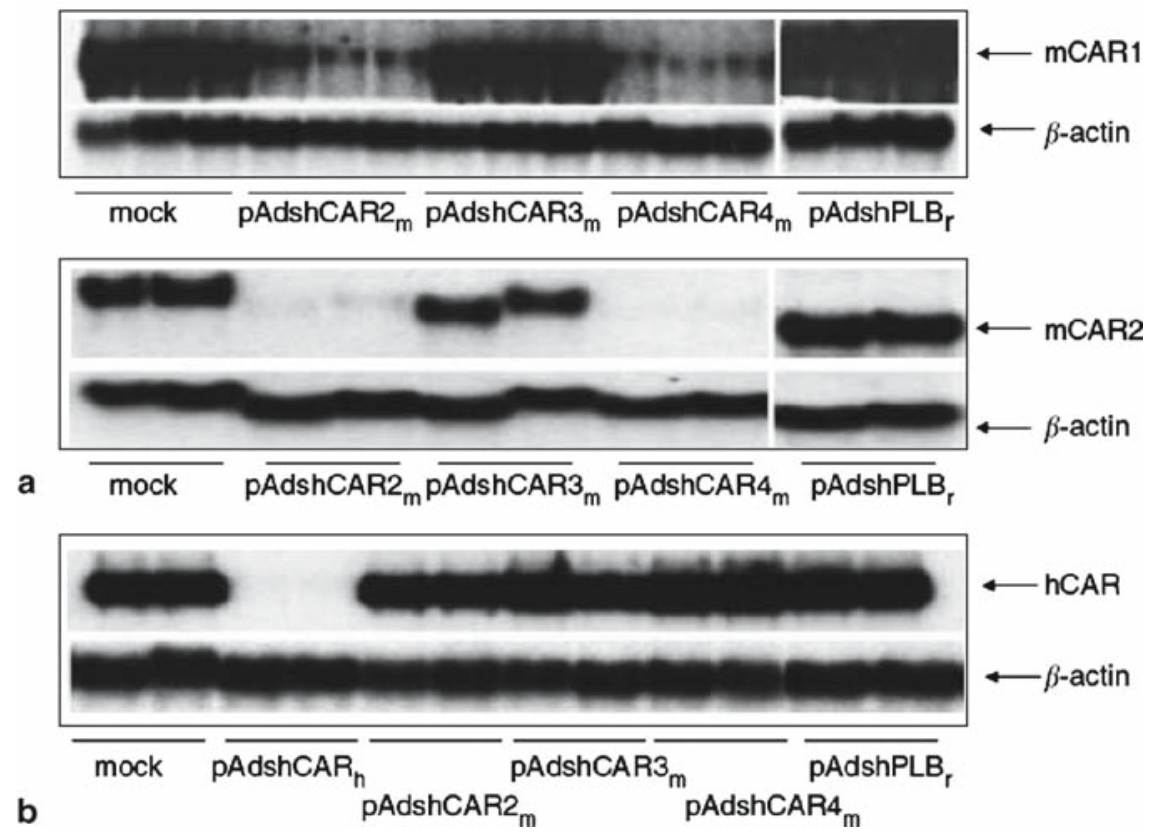

Fig. 3 Efficiency and specificity of shCARm-mediated mCAR silencing. (a) Selection of shCARm. 293T cells were co-transfected with mCAR1 (upper panel) or mCAR2 (lower panel) plus shCAR $2 \mathrm{~m}$, shCAR $3 \mathrm{~m}$, or shCAR4m expression plasmid. Northern blot analysis performed $48 \mathrm{~h}$ after transfection showed efficient downregulation of both mCAR1- and mCAR2-mRNA expression by shCAR $2 \mathrm{~m}$ and shCAR $4 \mathrm{~m}$ as compared to untransfected and control-shRNA (shPLBr) transfected cells. Experiments are made in triplicates (upper panel) and duplicates (lower panel). (b) Specificity of shCARm. 293T cells were co-transfected with human soluble CAR (hCAR) plus shCARm or human CAR-shRNA (pAdshCARh) expression plasmids. Northern blot analysis was performed as above. None of the shCARm downregulated hCAR, whereas human CAR-shRNA led to strong downregulation of hCAR-mRNA. Determination of $\beta$-actin expression was included as loading control. Experiments were made in duplicate

\subsubsection{Silencing of mCAR in Mouse Cardiac HL-1 Cells}

To determine the optimal AdV dose for transduction of mouse cardiac HL-1 cells, we first transduced HL-1 cells with a GFP-expressing marker AdV at an MOI from 5 to 1,000. At an MOI from 60 to 100 GFP expression was seen in 76-80\% of the cells, whereas no cytotoxic effects were observed. Further dose escalation resulted in an only slight further increase of transduction rate (Fig. 4a), and at an MOI of 500 or higher cytotoxic effects became evident (increase of apoptotic cells, reduction of cell growth). HL-1 cells express mCAR1 but no mCAR2 on their cell surface (not shown). Therefore, the silencing capacity of the new AdshCAR4m (Fig. 2) which expresses the above evaluated most efficient shCAR4m was investigated with respect to mCAR1 expression in HL-1 cells only. To assess the amount of AdV-generated shCAR4m, HL-1 cells were transduced with AdshCAR4m at an MOI from 5 to 1,000. Twenty-four, 48, and $72 \mathrm{~h}$ 
a

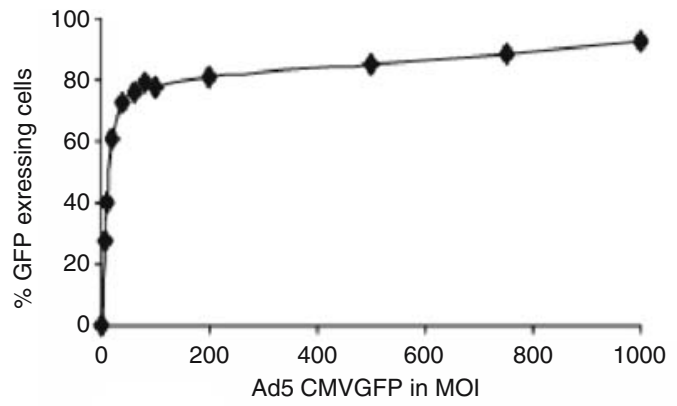

b
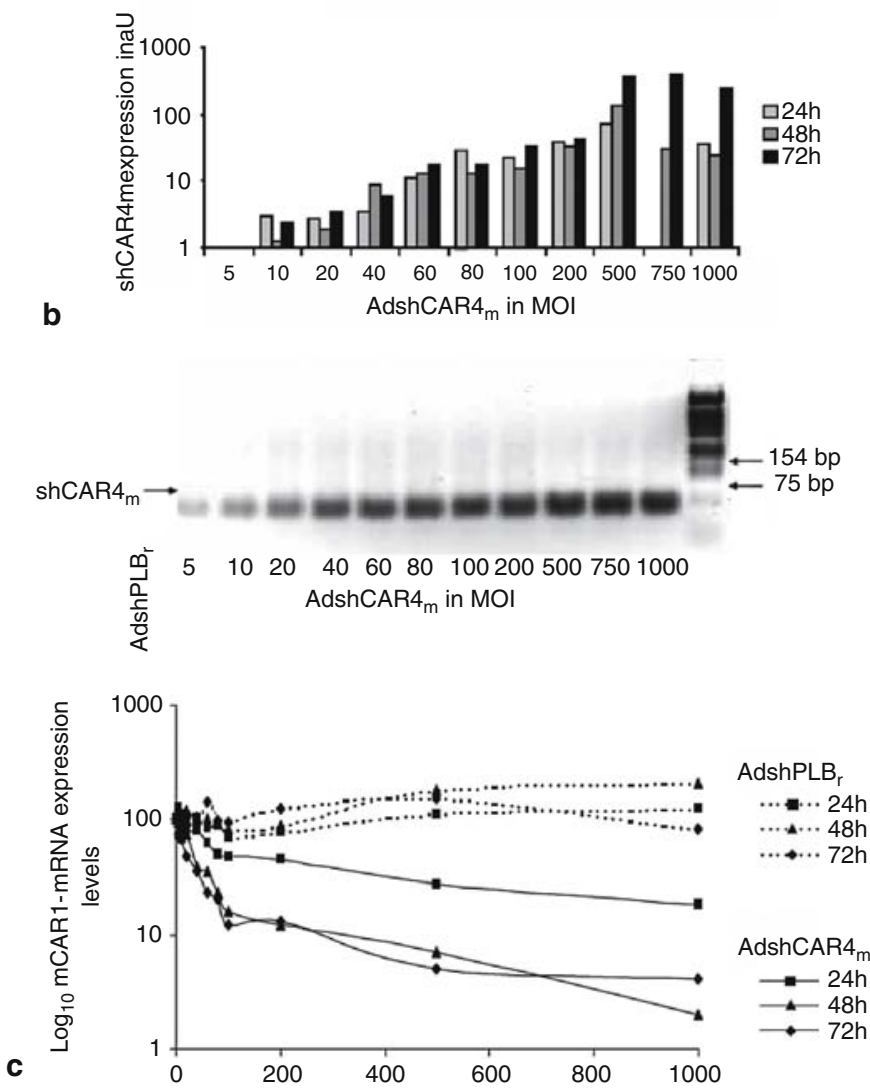

Fig. 4 Downregulation of mCAR mRNA in HL-1 cells by AdshCAR4m. (a) AdV-mediated gene transfer efficacy. HL-1 cells were transduced with Ad5CMVGFP (MOI from 5 to 1,000) and the fraction of cells expressing GFP was quantified by flow cytometry $48 \mathrm{~h}$ later. (b) AdV-mediated shCAR4m expression levels. HL-1 cells were transduced with AdshCAR4m (MOI from 5 to 1,000 ) and the shCAR4m expression level (shown as arbitrary units, aU) was then measured by real-time RT-PCR (upper panel) and agarose gel electorphoresis (lower panel, $24 \mathrm{~h}$ after transduction) at the indicated time points. HL-1 cells transduced with the vector AdshPLBr served as controls. (c) Downregulation of mCAR-mRNA expression by AdshCAR4m. HL-1 cells were transduced with AdshCAR4m or AdshPLBr (MOI from 5 to 1,000) and mCAR-mRNA expression was measured by real-time RT-PCR at the indicated time points 
later the expression of shCAR4m was analyzed. A time- and dose-dependent transcription of shCAR4m was detected (Fig. 4b). As a consequence of shRNA expression, strong downregulation of mCAR1-mRNA was observed 48 and $72 \mathrm{~h}$ after transduction. Near maximal reduction of $85-90 \%$ was observed at an MOI of 100. Higher doses did not result in significant further downregulation of mCAR1-mRNA (Fig. 4c). These results indicate that an MOI of 100 was optimal for HL-1 transduction, since this dose allowed strong downregulation of mCAR1-mRNA in HL-1 cells, while cytotoxicity of the viral vector was still very low.

We next investigated mCAR1 protein expression after transduction of HL-1 cells with AdshCAR4m at an MOI of 100. To disrupt pre-existing membrane-associated mCAR 1, HL-1 cells were trypsinized $24 \mathrm{~h}$ after AdshCAR4m transduction and then re-seeded. This procedure led to ablation of mCAR1 protein at the cell surface 2 days after transduction (Fig. 5a/b). In contrast, control cells rapidly re-expressed mCAR1 (Fig. 5b). Two days after AdshCAR4m treatment, a few cells still displayed some membrane-associated mCAR1 immunoreactivity (Fig. 5b), probably reflecting residual mCAR1 domains still anchored in the cell membrane after trypsinization. At days 3 and 4 after AdshCAR4m transduction mCAR1 immunoreactivity disappeared from HL-1 cells, whereas it remained permanently detectable in the control cells (Fig. 5b). Interestingly, CAR was only expressed at cell-to-cell contact sites in HL-1 cells suggesting that the observed stability of CAR in intact monolayers is determined by homophilic or heterophilic CAR interaction at cell-tocell contact sites. Together, the results indicate that AdshCAR4m treatment efficiently inhibits de novo synthesis of mCAR1.

\subsubsection{Inhibition of CoxB3 Replication in HL-1 Cells by AdshCAR4m}

The antiviral effect of mCAR1 silencing was now investigated with respect to CoxB3 for which cellular CAR constitutes the internalization receptor. For this purpose, we first investigated the CoxB3 protective efficacy of AdshCAR4m as a function of dose, at an MOI of AdshCAR4m ranging from 10 to 100, using the same trypsin-involved experimental procedure as described above. HL-1 cells were infected with CoxB3 $48 \mathrm{~h}$ after transduction with AdshCAR4m and the amount of new generated progeny virus determined six days later. Virus plaque assays clearly demonstrated that CoxB3 replicates in HL-1 cells, but at a rather low frequency, since no cytopathogenic effect was visible during the 6-day investigation period. As expected, the strongest reduction of CoxB3 progeny virus production by $97 \%$ was observed at the highest AdshCAR4m dose with an MOI of 100. Nevertheless, MOIs of 50 and 25 still led to significant inhibition of CoxB3 replication by 85 and $75 \%$, respectively, whereas a MOI of 10 showed no significant effect (Fig. 6a). To investigate whether disruption of pre-existing CAR affects CoxB3 infection we treated HL-1 cells with the trypsin-involved experimental procedure as described above or omitted the trypsin step after AdshCAR4m transduction. Titers of CoxB 3 progeny virus generated 5 and 6 days after CoxB3 infection were strongly reduced by about $97 \%$ in AdshCAR4m-transduced cells, 


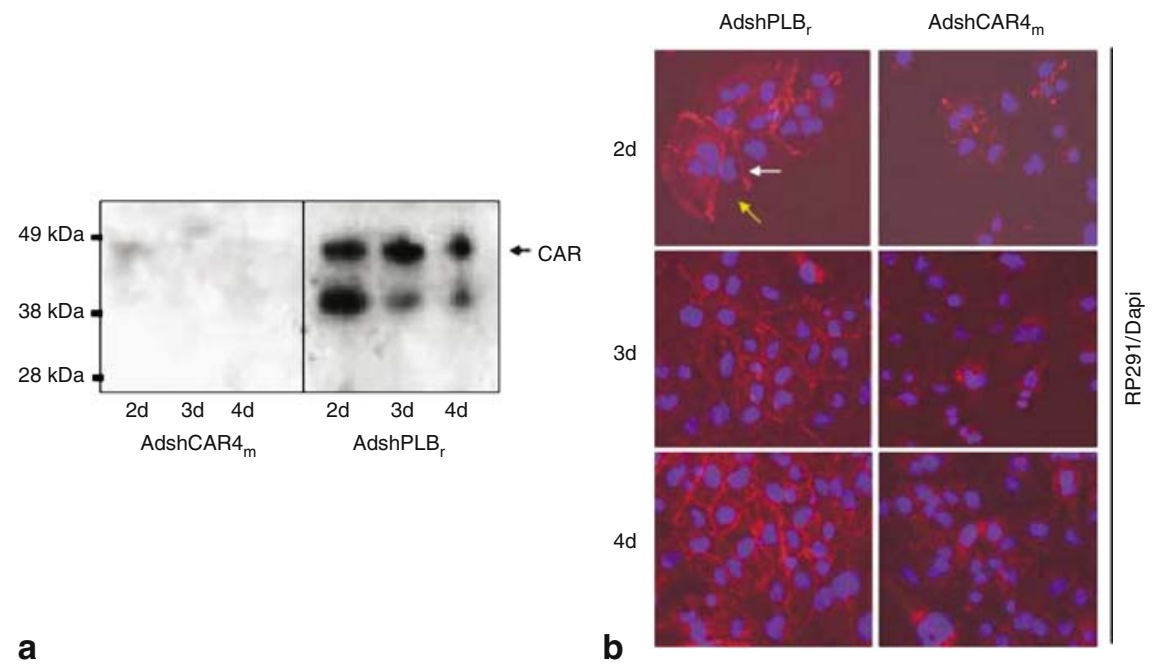

Fig. 5 Downregulation of mCAR1 protein in HL-1 cells by AdshCAR4m. (a) Detection of mCAR1 ablation by Western blot analysis. HL-1 cells were transduced with AdshCAR4m or the control vector AdshPLBr (MOI of 100) for 24h. Cells were then trypsinized, re-seeded, and investigated using the polyclonal rabbit-anti-CAR antibody H-300 directed against the extracellular domain of CAR. mCAR1 protein became undetectable as early as 2 days after transduction with AdshCAR4m, whereas in the controls mCAR1 protein was abundantly expressed. (b) Detection of mCAR1 knockdown by indirect immunofluorescence. HL-1 cells were treated as in (a). mCAR1 expression was detected by indirect immunofluorescence using the polyclonal rabbitantibody RP-291 (Sollerbrant et al. 2003) directed against the CAR variant with intracellular SIV tail (mCAR variant 1, accession no. NM_001025192). mCAR1 was abundantly expressed over the whole investigation period (4 days after transduction) in HL-1 cells transduced with control vector (left-hand panels). In contrast, mCAR1 was visible at low levels 2 days after transduction but became undetectable at later time points (right-hand panels). Cell nuclei are stained with DAPI and overlayed images are shown. Notably mCAR1 was localized only at cell-cell contact sites (white arrows), whereas cell membrane regions not in contact with other cells displayed no mCAR1 protein (yellow arrows). mCAR2 expression was not detectable in HL-1 cells by using polyclonal rabbit-antibody RP-194 [56] directed against the CAR variant with intracellular TVV tail (mCAR variant 2, accession no. NM_009988) (not shown)

irrespective of whether membrane-associated CAR was disrupted by trypsinization or not (Fig. 6b). These experiments indicate that AdshCAR4m inhibits CoxB3 replication in HL-1 cells with high efficacy. Moreover, proteolysis of membrane-associated CAR seems to be not affect CoxB3 infection.

\subsubsection{Inhibition of Adenovirus Infection of HL-1 Cells by AdshCAR4m}

CAR is also involved in adenovirus infection as attachment receptor. To investigate the long-term efficacy of AdshCAR4m with respect to the inhibition of 


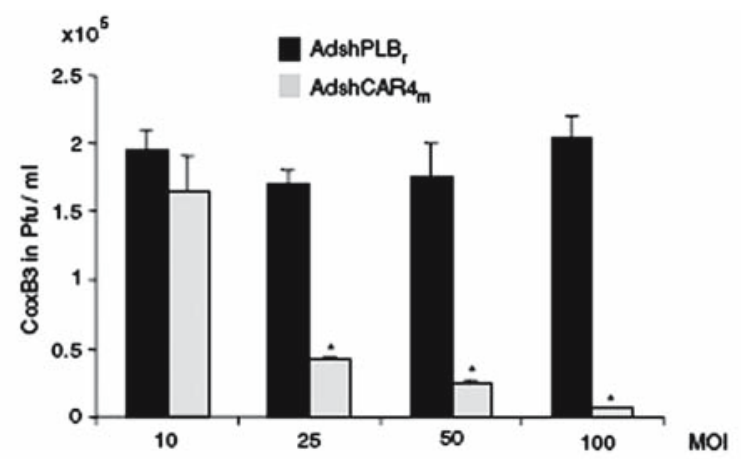

a

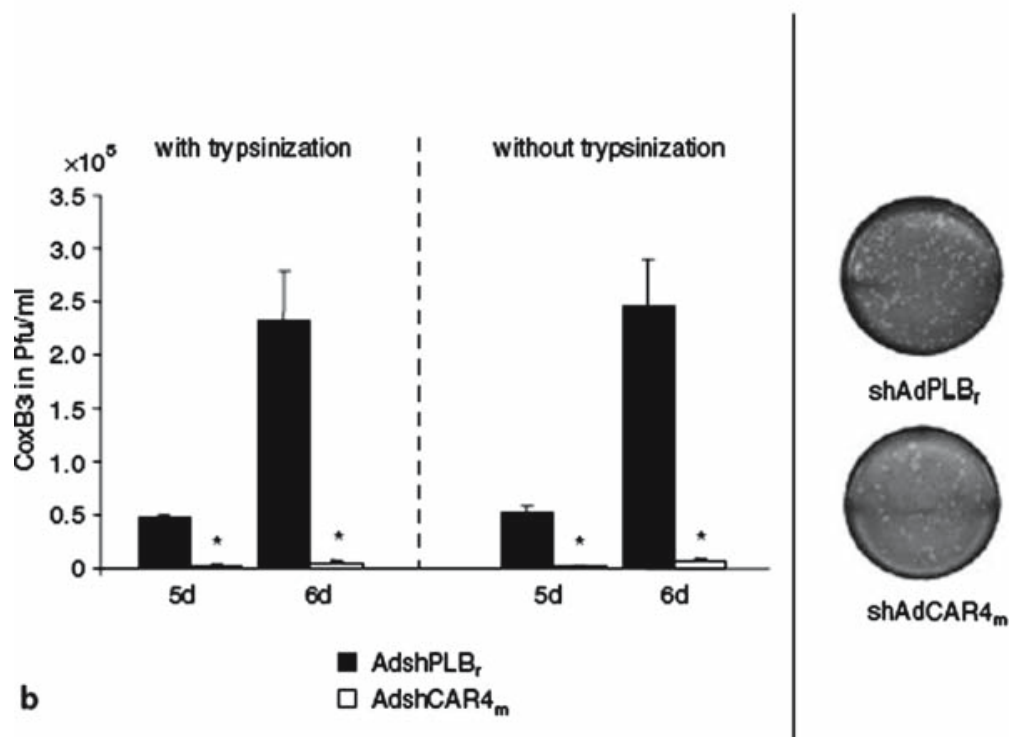

Fig. 6 Inhibition of CoxB3 infection by mCAR silencing in HL-1 cells. (a) Inhibition of CoxB3 replication as a function of AdshCAR4m dose. HL-1 cells were transduced with AdshCAR4m or control vector at variable MOIs ranging from 10 to 100 . The cells were trypsinated $24 \mathrm{~h}$ later, re-seeded and infected with with CoxB3 (at an MOI of 1) 24h later. Plaque assays were carried out 6 days post infection. Asterisks indicate significant differences $(p<$ 0.05). (b) Inhibition of CoxB3 replication by AdshCAR4m as a function of trypsin treatment. HL-1 cells were transduced with AdshCAR4m or control vector (MOI of 100) as in Fig. 5. 24h later cells were trypsinated and re-seeded (with trypsinization) or medium was changed (without trypsinization). $48 \mathrm{~h}$ after transduction the cells were infected with CoxB3 at an MOI of 1. Five and six days later cells were lysed by three freeze/thaw cycles and virus titers were determined by plaque assay on HeLa cells (left-hand diagram). Right side Example for plaque assay on HeLa cells using a dilution of 1:500 of HL-1 cell lysate from AdshCAR4m and control vector transduced cells 

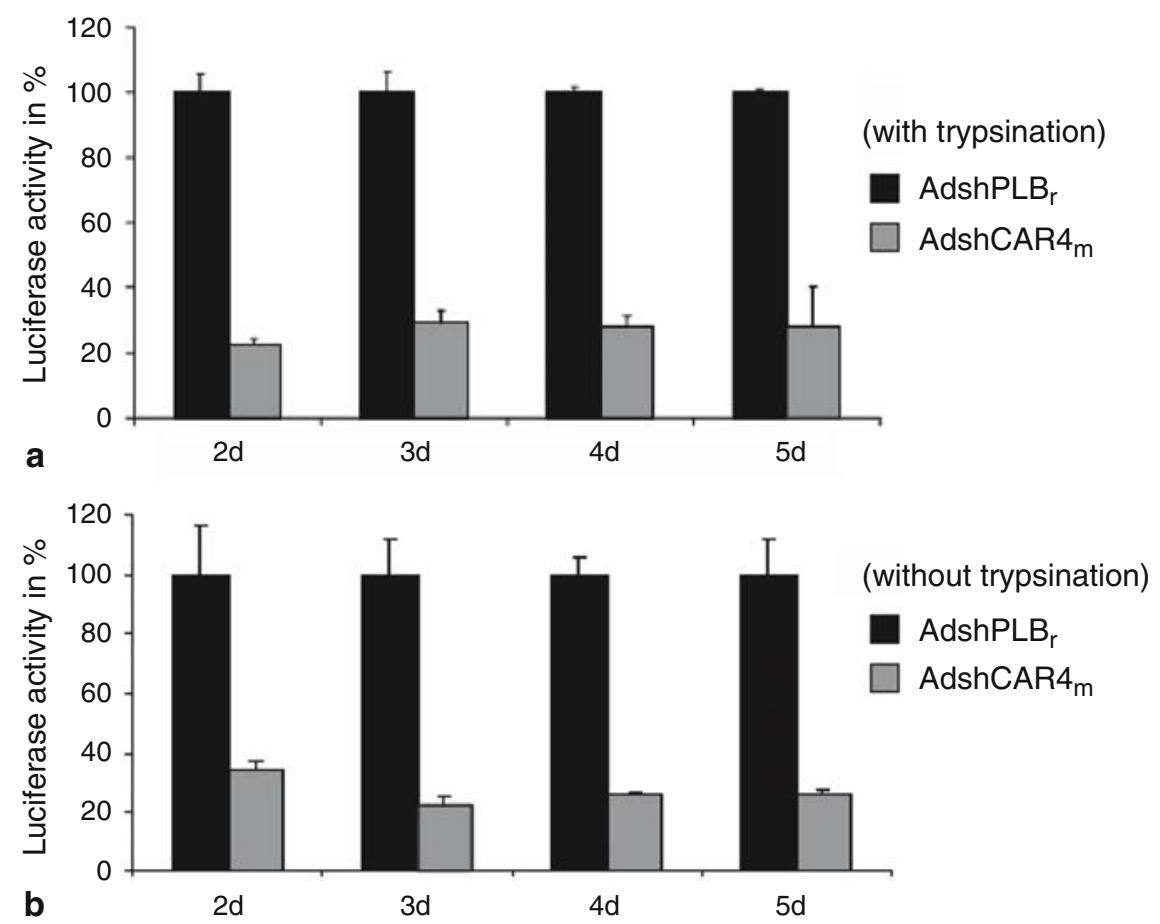

Fig. 7 Inhibition of adenovirus infection by mCAR silencing in HL-1 cells. (a) Inhibition of adenovirus infection by AdshCAR4m (with trypsinization). HL-1 cells were treated as in Fig. 5 . Two, three, four, and five days after transduction the cells were infected with a luciferase marker adenovirus (MOI of 5) and luciferase expression was measured after $24 \mathrm{~h}$. (b) Inhibition of adenovirus infection by AdshCAR4m (without trypsiinization). HL-1 cells were transduced with AdshCAR4m or the control vector AdshPLBr (each MOI of 100). The medium was changed after $24 \mathrm{~h}$. Two, three, four, and five days after transduction the cells were infected with a luciferase marker adenovirus (MOI of 5) and luciferase expression was measured 24h later

adenovirus infection and to investigate whether proteolysis of pre-existing CAR affects adenovirus infection, HL-1 cells were transduced with AdshCAR4m versus the control vector at an MOI of 100. Twenty-four hours later, cells were either trypsinized and re-seeded, or the medium was changed (treatment without trypsinization). At 2, 3, 4 and 5 days after transduction, the cells were infected with a luciferase marker adenovirus (MOI of 5). The measurement of luciferase activity $24 \mathrm{~h}$ later revealed persistent reduction of marker gene expression of about $\approx 75 \%$ in AdshCAR4m-treated HL-1 cells during the 5-day investigation period. This clearly demonstrates that CAR-shRNA treatment inhibits adenovirus uptake into HL-1 cells, but markedly less efficient than for CoxB3. Inhibition of adenovirus infections was closely similar between trypsinized and non-trypsinized cells (Fig. 7) indicating that proteolysis of CAR does not affect the function of CAR in adenovirus infections. 


\subsubsection{The Anti-Adenovirus and Anti-CoxB3 Effect of CAR Silencing in PNCMs}

As HL-1 cells represent a permanent tumor cell line from heart, we were also interested in investigation of the effect of CAR silencing onto viral infection in primary cardiac cells. For this reason, PNCMs were transduced with AdshCAR2m, which shRNA sequence complete matches with mouse and rat CAR sequences or the control vector AdshGFP, both at an MOI of 100. At this dose nearly $80 \%$ of PNCMs are transduce with AdV (results not shown), which is closely similar to the HL-1 cells. Following treatment of PNCMs with AdshCAR2m CAR expression was unaffected up to day 3 after transduction, but it becomes strongly downregulated 6 days and 8 days after transduction (Fig. 8a). As a consequence of delayed CAR downregulation challenge of AdshCAR2m-treated PNCMs with CoxB3 (MOI of 1) resulted in delayed inhibition of CoxB3 replication. In fact, significant reduction of CoxB3 replication was seen if PNCMs were infected with $\mathrm{CoxB} 3$ at day 6 and 8 but not at day 3 after AdshCAR2m transduction. Moreover, inhibition of CoxB3 replication strongly depended on the initially used AdshCAR2m dose. An MOI of 100 of AdshCAR2m resulted in reduction of CoxB3 titers of about 66 and $90 \%$, while an MOI of 25 resulted in reduction of CoxB3 titers of only 16 and $34 \%$ at day 6 and 8, respectively (Fig. 8b). Very similar results were obtained if AdshCAR2m (MOI 100) transduced PNCMs were infected with AdVs. Adenovirus uptake was reduced of about $85 \%$ at day 6 and about $92 \%$ at day 8 after AdshCAR2m transduction, while no effect was seen at day 3 (Fig. 8c).

\subsection{Discussion of Anti-Viral Strategies}

CoxB3 and adenoviruses are common agents of acute myocarditis and inflammatory cardiomyopathy (Bowles et al. 1986; Kühl et al. 2005a). Although interferon- $\beta$ treatment has the potential to eliminate cardiotropic viruses and to improve heart function in patients with myocardial persistence of viral genomes (Kühl et al. 2003), no specific treatment against CoxB3 and adenovirus infections is available to date. With respect to antiviral therapy, RNAi is a promising new technology. Its therapeutic potential against viral infections has been demonstrated in multiple in vitro and in vivo studies (Bitko et al. 2005; McCaffrey et al. 2003; Carmona et al. 2006; Park et al. 2003; Merl et al. 2005; Werk et al. 2005). Despite encouraging results, however, several reports have revealed a fundamental general problem of siRNA-based antiviral therapy. Due to the requirement for strict sequence-specificity between therapeutic siRNA and target mRNA, even single point mutations in the viral target sequence may result in the rapid selection of escape mutants during persistent viral infections (Wilson and Richardson 2005; Boden et al. 2007; Gitlin et al. 2005).

In this study, we show that inhibition of CoxB3 and adenovirus infections is possible via silencing of the CAR receptor by means of vector-generated CAR-shRNA. This strategy is an attractive alternative to siRNA-based silencing of virus-encoded gene, since it drastically reduces the probability of escape mutant development (Arrighi et al. 


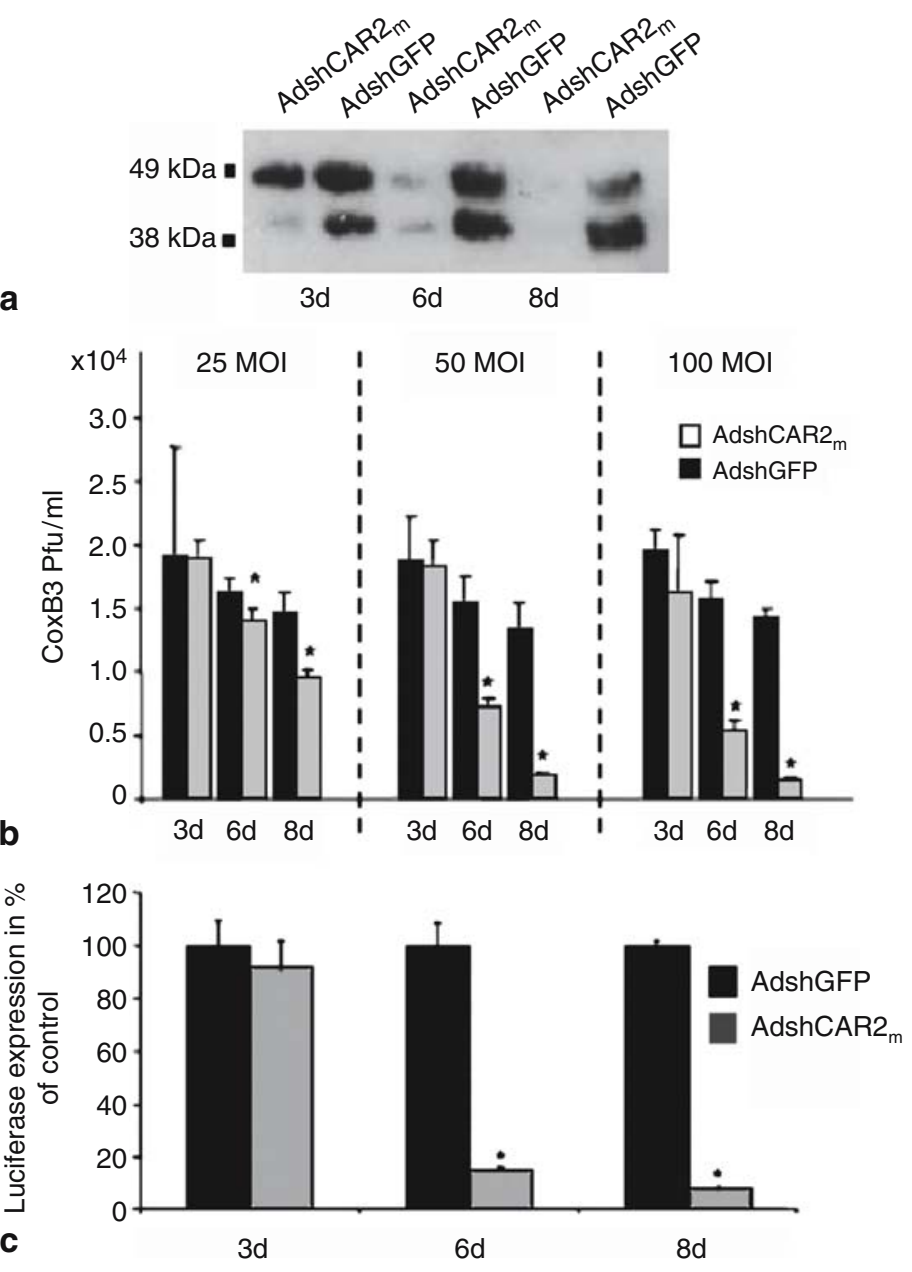

a

Fig. 8 Inhibition of CoxB3 and adenovirus infection in PNCMs by CAR silencing. (a) Detection of rat CAR downregulation by Western blot analysis. PNCM's were transduced with AdshCAR2m or the control vector AdshGFP (each MOI of 100) for 3, 6, and 8 days and than investigated for CAR expression using the polyclonal rabbit-anti-CAR antibody H-300. (b) PNCMs were transduced with AdshCAR2m or the control vector AdshGFP (MOI of 25, 50 and 100) for 3, 6 and 8 days and then infected with CoxB3 (MOI of 1). Cells were harvested 3 days later and CoxB3 plaque titers determined by plaque assay. Asterisks indicate significant differences $(p<0.05)$. (c) PNCMs were transduced with AdshCAR2m or the control vector AdshGFP (each at MOI of 100) for 3, 6 and 8 days and then transduced with a luciferase marker adenovirus (MOI of 5). Luciferase expression was measured $24 \mathrm{~h}$ later. Asterisks indicate significant differences $(p<0.005)$

2004). In contrast to previous work (Werk et al. 2005), our investigations were carried out in myocardial cells representing the actual targets for CoxB3 and adenovirus infections in acute myocarditis and inflammatory cardiomyopathy (Kandolf 2004). Treatment of HL-1 cells with the shCAR vector led to efficient and stable removal of 
mCAR protein from the cell surface and consequently, to significant inhibition of CoxB3 and adenovirus infection of these cells. Remarkably, adenovirus internalization was reduced by only $\approx 75 \%$ despite near complete silencing of mCAR expression, while CoxB3 infection was inhibited by up to $97 \%$. In PNCMs, however, CAR silencing resulted in up to 92 and $90 \%$ inhibition of adenovirus and CoxB3 infection, respectively. Adenovirus binding to cells is not exclusively mediate by CAR. Type 2 and 5 adenoviruses use CAR to attach to the cell surface (Bergelson et al. 1997), while its internalization occurs through $\alpha v \beta 1, \alpha v \beta 3$, and $\alpha v \beta 5$ integrin (Tomko et al. 1997; (Wickham et al. 1993; Li et al. 2001). Others have shown that heparan sulfate glycosamino-glycans and MHC class I molecules may also be involved in the binding of adenovirus 2 and 5 (Hong et al. 1997; (Dechecchi et al. 2001). Moreover, expression of receptors involved in adenovirus infection is highly variable between different cell types and tissues (Fechner et al. 1999). Thus, variable receptor expression levels and interaction of adenoviruses with cell type specific expressed alternative receptors is the most likely explanation for the failure of complete CAR silencing to achieve efficient blockade of adenovirus infection in HL-1 cells. To enhance efficacy in inhibition of adenovirus infection via the anti-receptor approach, therefore, it seems to be necessary to simultaneously inhibit CAR and its co-receptors.

In contrast to the anti-adenovirus experiments, the anti-CAR approach was efficient with respect to CoxB3 in both HL-1 cells and PNCMs. The magnitude of inhibition of CoxB3 infection correlated well with the vector-induced ablation of mCAR from the cell surface and emphasizes the key role of CAR for CoxB3 infections of cardiac cells. The molecular reason for the differences of anti-CAR treatment in adenovirus versus CoxB3 infections may be due to the unique role playing CAR during $\mathrm{CoxB} 3$ infection as it induces conformational changes in the virus capsid that are essential for CoxB3 entry into the target cell (Coyne and Bergelson 2006). The high efficacy of the shCAR vector against this virus indicates, that CAR silencing is a promising therapeutic approach against CoxB3 infections of the myocardium. Previous studies have shown a similar degree of inhibition when using siRNAs directed against CoxB3 viral genomic RNA or its RNA intermediate (Merl et al. 2005; Werk et al. 2005; Yuan et al. 2005). Combination of direct anti-viral approaches which are prone to escape mutant development with the current anti-receptor strategy are likely to further increase the efficacy of RNAi-based antiviral therapy.

Recently, it has been shown that silencing of CAR by chemically synthetisized siRNA inhibited CoxB3 infection in human cells of non-cardiac origin (Werk et al. 2005). However, this approach decreased virus titers by only $\approx 60 \%$ which is markedly less than in the present study employing AdV-generating shCAR. This difference should be the result of high transduction efficacy of AdVs and the prolonged high-level expression of the shCAR. A bottleneck of chemically synthesized siRNAs is its transient silencing activity of $\approx 3$ days in cardiac cells (Watanabe et al. 2004). Viral vectors expressing shRNAs, however, enable long term silencing of target genes in cells of cardiac origin (Fechner et al. 2007) and allow efficient delivery of transgenic sequences to the heart (Ikeda et al. 2002; Wang et al. 2005). Between them, pseudotyped AAV2/9 vectors seems to be most promising vectors for cardiac gene transfer as they enable 
highly efficient transduction of the heart by use of the simple intravenous application route (Pacak et al. 2006; Inagaki et al. 2006).

CAR is a cell adhesion protein mediating homotypic (Cohen et al. 2001; Honda et al. 2000) and heterotypic (Zen et al. 2005) intercellular interactions. It is a component of specialized intercellular junctions including epithelial tight junctions (Cohen et al. 2001), neuro-muscular junctions (Shaw et al. 2004), and myocardial intercalated discs (Noutsias et al. 2001). We found it to be localized exclusively at cell-to-cell contact sites between HL-1 cells, possibly as a consequence of cell membrane-associated CAR stabilization through homotypic (CAR-CAR) or heterotypic (CAR-protein) intercellular protein interactions. In contrast to previous CAR silencing attempts using synthetic siRNAs, the newly developed viral vectors AdshCAR4m and AdshCAR2m achieved near complete and stable knockout of CAR. This was an essential prerequisite to reveal significantly different kinetics of CAR protein ablation in different cell types, in particular an unexpectedly high stability of CAR in primary cells versus a stable cell line. These data may be explained by cell type-specific CAR protein kinetics. But, differences in CAR mRNA stability or processing of the CAR-shRNA through the RNA interference machinery also have to be taken into account. The delayed downregulation of CAR following vector-mediated CAR-shRNA delivery into primary cardiomyocytes is relevant for in vivo investigations, since the time lag between vector application and CAR silencing needs to be taken into consideration in antiviral therapy studies.

One important concern associated with downregulation of a cellular receptor molecule for antiviral therapy is possible side effects resulting from ablation of the normal cellular functions of the receptor. During organogenesis, CAR is highly expressed in the heart, but rapidly downregulated post partum (Fechner et al. 2003a). In a recently published genomic mouse knockout model, cardiomyocyte-specific CAR deletion resulted in severe cardiac anomalies and death between day 11.5 and 13.5 of embryonal development (Chen et al. 2006; Dorner et al. 2005). If the CAR gene became ablated late in embryonic development, however, the CAR-deficient animals survived into adulthood and had no evident cardiac anomalies (Chen et al. 2006). This finding suggests that CAR silencing by RNAi as employed in our study should be well tolerable over considerable periods of time, during which virus migration and spreading were efficiently inhibited. Furthermore, treatment of HL-1 cells with AdVgenerating shCAR revealed no side effects since we found no changes in cell growth, cell morphology, or F-actin cytoskeleton protein expression (not shown). However, as $\mathrm{CAR}$ is an integral component of the tight junctions and may play an important role in cell methabolism in several tissues and organs, experiments have to confirm that shRNA-mediated CAR knockdown does not have undesirable side effects in vivo.

In summary, high efficacy of CAR gene silencing by shRNAs in cardiac-derived cells was observed against CoxB3, while variable results were obtained for adenoviruses. In contrast to previous silencing attempts using siRNAs or shRNA-plasmid the newly developed shRNA-vectors were able to achieve near complete and stable knockout of CAR transcription. This in turn revealed significantly different responses of cellular CAR protein to CAR-shRNA treatment which need to be considered as important determinants of this new antiviral approach in vivo. 


\section{RNA Interference to Improve Cardiac Function in Advanced Heart Failure}

\subsection{Overview}

Impaired function of the phospholamban (PLB)-regulated sarcoplasmic reticulum (SR) $\mathrm{Ca}^{2+}$ pump (SERCA2a) contributes to cardiac dysfunction in heart failure (HF). PLB downregulation may increase SERCA2a activity and improve cardiac function. Small interfering (si)RNAs mediate efficient gene silencing by RNA interference (RNAi). However, their use for in vivo gene therapy is limited by siRNA instability in plasma and tissues, and by low siRNA transfer rates into target cells. To address these problems we developed an adenoviral vector (AdV) transcribing short hairpin (sh)RNAs against rat PLB and evaluated its potential to silence the PLB gene and to modulate SERCA2a-mediated $\mathrm{Ca}^{2+}$ sequestration in primary neonatal rat cardiomyocytes (PNCMs). Over a period of 13 days, vector transduction resulted in stable $>99.9 \%$ ablation of PLB-mRNA at an MOI of 100 . PLB protein gradually decreased until day 7 ( $7 \pm 2 \%$ left), whereas SERCA, $\mathrm{Na}^{+}$/ $\mathrm{Ca}^{2+}$ exchanger (NCX1), calsequestrin (CSQ2), and troponin I (TnI) protein remained unchanged. PLB silencing was associated with a marked increase in ATPdependent oxalate-supported $\mathrm{Ca}^{2+}$ uptake at $0.34 \mu \mathrm{M}$ of free $\mathrm{Ca}^{2+}$, and rapid loss of responsiveness to PKA-dependent stimulation of $\mathrm{Ca}^{2+}$ uptake was maintained until day 7. In summary, these results indicate that AdV-derived PLB-shRNA mediates highly efficient, specific, and stable PLB gene silencing and modulation of active $\mathrm{Ca}^{2+}$ sequestration in PNCMs. The availability of the new vector now enables employment of RNAi for the treatment of HF in vivo.

\subsection{Regulatory RNA- and Gene-Based Therapies of Heart Failure}

Heart failure (HF) remains a leading cause of mortality in the developed world. Deteriorated function of the failing heart has been partially attributed to dysfunction of the PLB-controlled SERCA2a (Piacentino et al. 2003). Reduction of both SERCA2a expression and PLB phosphorylation (Wolska et al. 2002; Schmidt et al. 1999) may contribute to this dysfunction. Nonphosphorylated PLB keeps the $\mathrm{Ca}^{2+}$ affinity of SERCA2a low resulting in decreased SR $\mathrm{Ca}^{2+}$ uptake, slowed relaxation and decreased SR $\mathrm{Ca}^{2+}$ load, while PLB phosphorylation in response to $\beta$-adrenergic stimulation relieves this inhibition. Germline transgenic approaches for ablation of PLB expression and function in mice (Luo et al. 1994), and somatic gene transfer for dominant negative PLB mutants (Iwanaga et al. 2004; Hoshijima et al. 2002), PLB-antisense-RNAs (Eizema et al. 2000; Li et al. 2005; He et al. 1999; delMonte et al. 2002), or intracellular inhibitory PLB antibodies (Dieterle et al. 2005; Meyer et al. 2004) were employed to increase cardiac SR Ca ${ }^{2+}$ transport activity and hence 
the contractile function of cardiomyocytes under physiological and diseased conditions. RNA interference (RNAi) mediated by chemically synthesized siRNAs was recently employed to silence PLB expression in cardiomyocytes (Watanabe et al. 2004). RNAi relies on post-trancriptional, sequence-specific gene silencing via small homologous double-stranded RNAs (Leung and Whittaker 2005). Its silencing efficacy is higher than that of antisense RNAs (Ogorelkova et al. 2006). Nevertheless, therapeutic use of synthetic siRNAs is significantly limited by their rapid degradation in target cells, resulting in only transient gene silencing (Watanabe et al. 2004), and by the difficulties in achieving sufficient transfer rates into multiple cells of therapeutic interest including cardiomyocytes. These limitations are significantly aggravated in vivo and despite intense efforts in recent years systemic delivery of siRNAs remains a major hurdle for in vivo applications of RNAi (Lewis et al. 2002). Since viral vector systems have been shown to be suitable to overcome these limitations in vitro and in vivo, we have developed a novel adenoviral vector (AdV) suitable for transcription of shRNA targeting PLB. Treatment with this vector resulted in highly efficient and specific PLB gene silencing in primary neonatal rat cardiac myocytes (PNCMs) which was stable over 2 weeks and associated with a marked increase in the SERCA2a-catalyzed $\mathrm{SR} \mathrm{Ca}^{2+}$ sequestration.

\subsection{RNAi-Based Modulation of Cardiac $\mathrm{Ca}^{2+}$ Homeostasis in Heart Failure}

In order to select potential PLB-siRNA sequences for the generation of PLB-shRNAs, we initially co-transfected Cos-7 cells with an GFP-rat PLB fusion construct plus different siRNAs directed against rat PLB. Among five tested siRNAs, three were highly efficient in downregulating the fusion transcripts. The most efficient of these siRNAs was then cloned into a shRNA expression plasmid, as a DNA sequence encoding the PLB-shRNA. The co-transfection experiments with the GFP-rat PLB fusion constructs showed the very high efficacy of PLBshRNA17 (Fig. 9). Based on these results we constructed an AdV designated AdshPLBr which generates PLB-shRNA17 (Fig. 10). To assess the efficacy of the vector AdshPLBr in downregulating endogenously expressed PLB in the actual target cells, we transduced primary neonatal rat cardiomyocytes (PNCMs) with AdshPLBr and investigated the dose- and time-dependency of its action. A control AdV (AdshCAR4m) generating an shRNA irrelevant for PLB and cardiac $\mathrm{Ca}^{2+}$ metabolism was employed to test for target specificity of AdshPLBr. The treatment of PNCMs with AdshPLBr resulted in strong dose-dependent downregulation of PLB-mRNA expression, by $27 \%$ at an MOI of 10 and by $87 \%$ at an MOI of 100, 2 days after transduction (Fig. 11a). Rapid, strong, and enduring PLB silencing was observed after treatment of PNCMs with AdshPLBr (MOI = 100) over a prolonged 13-day investigation period. PLB-mRNA abundance was extremely low $<0.1 \%$ of baseline as quantitated by real-time-RT-PCR (not shown) as early as day 1 and remaining at this level until day 13 (end of experiment). By 

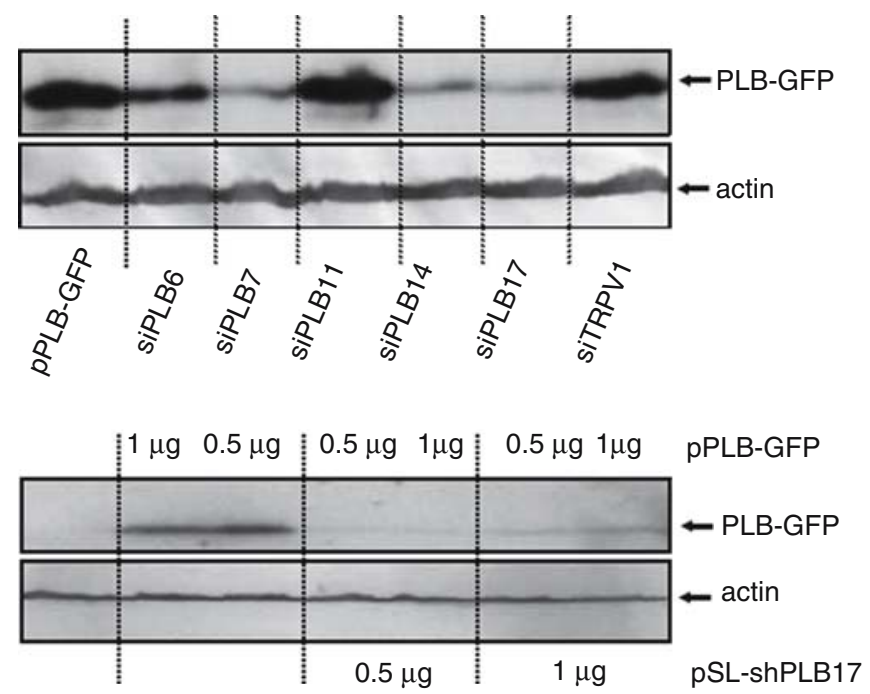

Fig. 9 Evaluation of phospholamban (PLB) siRNAs. Upper Selection of PLB siRNAs. Cos-7 cells were co-transfected with the PLB-GFP fusions construct pPLB-GFP and synthetic PLBsiRNAs. Cells were harvested $20 \mathrm{~h}$ later and western-blot carried out. PLB-GFP fusion protein was detected by a rabbit antiserum against GFP. To confirm equal loading of the samples, membranes were stripped and reprobed with a monoclonal antibody against actin. Note: siTRPV1 is a synthetic siRNA directed against the vanilloid receptor 1 (TRPV1). Lower Dose-dependency of pSLshPLB17 action. Cos-7 cells were co-transfected with the pSL-shPLB17 and pPLB-GFP as indicated. Immunoblots were carried out $24 \mathrm{~h}$ after transfection as described above

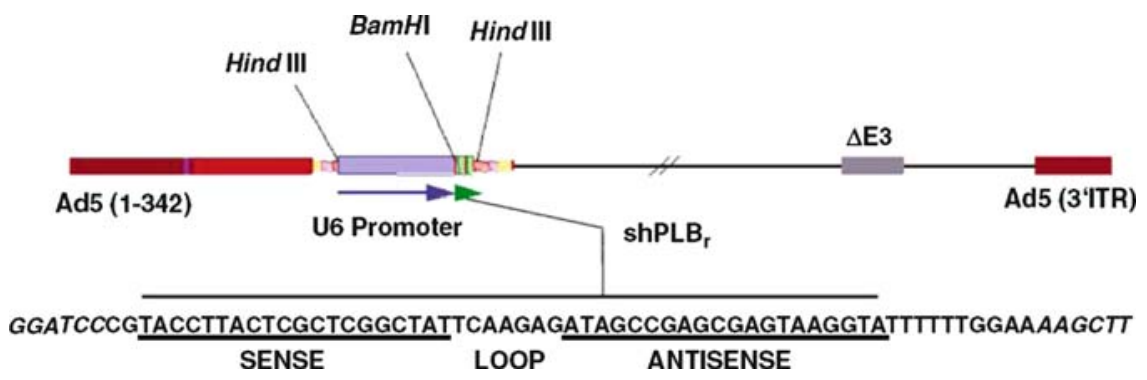

Fig. 10 Map of AdshPLBr vector and PLB-shRNA sequence

contrast, PLB-mRNA expression stayed at baseline level both in non-transduced controls and AdshCAR4m-treated PNCMs (Fig. 11b). Similar but delayed changes occurred at the PLB protein level. A decrease in PLB protein became first visible on day 3 (51 $\pm 4 \%$ remaining) as compared to controls. PLB protein then further decreased continuously until day 7 ( $7 \pm 2 \%$ remaining). By contrast, the protein levels of SERCA2, NCX1, TnI, and CSQ2 remained unchanged indicating the absence of unspecific side effects on these cardiac proteins of both the 


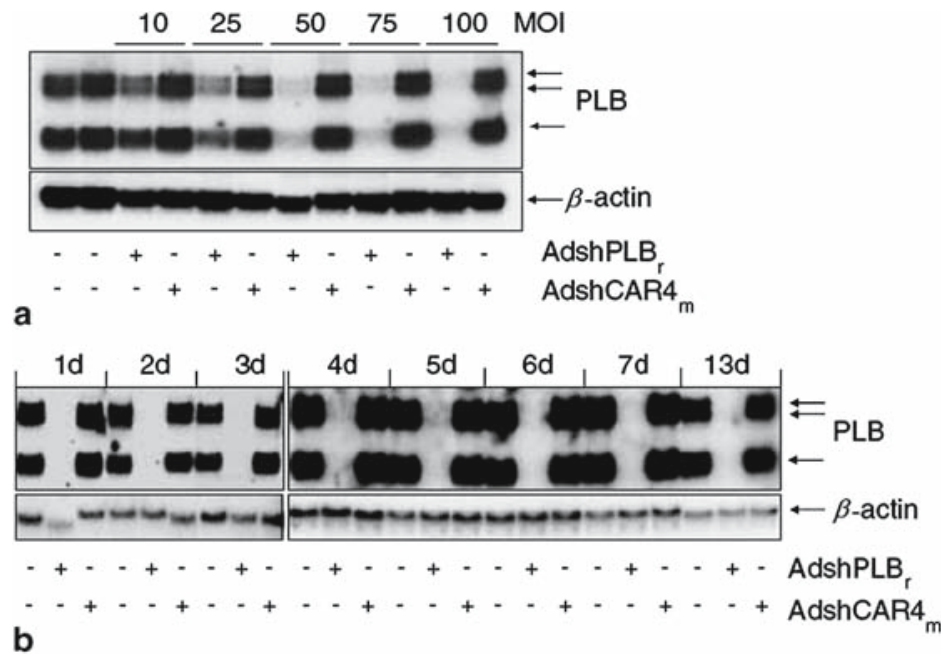

Fig. 11 Silencing of PLB-mRNA expression by AdshPLBr-generated PLB-shRNA. (a) Dosedependency of AdshPLBr-mediated PLB-mRNA downregulation. PNCMs were transduced with AdshPLBr or a control vector as indicated. Cells were harvested $48 \mathrm{~h}$ later and Northern-blot carried out using a rat PLB specific probe. To confirm equal RNA, loading blots were striped and rehybridized with an $\alpha$-actin specific probe. (b) Time-dependency of AdshPLBr-mediated PLBmRNA downregulation. PNCMs were transduced with AdshPLBr or a control vector at an MOI of 100. Cells were harvested at indicated time points after transduction and Northern-blot carried out as described above. Note: PLB-mRNA was undetectable during the complete 13 days investigation period in PNCMs transduced with AdshPLBr while its expression stayed unaffected in cells transduced with the control vector

vector itself and of the shRNA that it generates (Fig. 12a/b). To examine the functional consequences of the PLB-shRNA-induced decline in the PLB protein level, PNCMs were transduced with AdshPLBr or control vector AdshCAR4m (each at an MOI of 100). Cell homogenate $\mathrm{SR} \mathrm{Ca}^{2+}$ uptake rates were determined at submicromolar $(0.34 \mu \mathrm{M})$ and saturating $(3.68 \mu \mathrm{M})$ free $\mathrm{Ca}^{2+}$ concentrations. At the latter, the SR $\mathrm{Ca}^{2+}$-ATPase is known to be insensitive to non-phosphorylated PLB, whereas at submicromolar $\mathrm{Ca}^{2+}$ non-phosphorylated PLB has been shown to decrease the $\mathrm{Ca}^{2+}$ affinity of this enzyme 17 . The rate of $\mathrm{Ca}^{2+}$ uptake determined at $0.34 \mu \mathrm{M}$ free $\mathrm{Ca}^{2+}$ and normalized to the maximum uptake rate (Vmax) values at saturating $\mathrm{Ca}^{2+}$ (relative $\mathrm{Ca}^{2+}$ uptake rate) did not differ between non-transduced and AdshCAR4m- or AdshPLBr-treated PNCMs one day after transduction day 1 (approximately $45 \%$ of $\mathrm{Vmax}$ each). The relative $\mathrm{Ca}^{2+}$ uptake rate remained at this level both in non-transduced and AdshCAR4m-transduced PNCMs until day 7 after transduction. In contrast, it increased steadily in AdshPLBr-transduced PNCMs reaching 100\% of Vmax on day 5 after transduction and remaining at this high level until the end of the experiment on day 7 (Fig. 13a). This indicates an increase in the $\mathrm{Ca}^{2+}$ affinity of the $\mathrm{SR} \mathrm{Ca}^{2+}$-ATPase in the PLB-deficient PNCMs. 

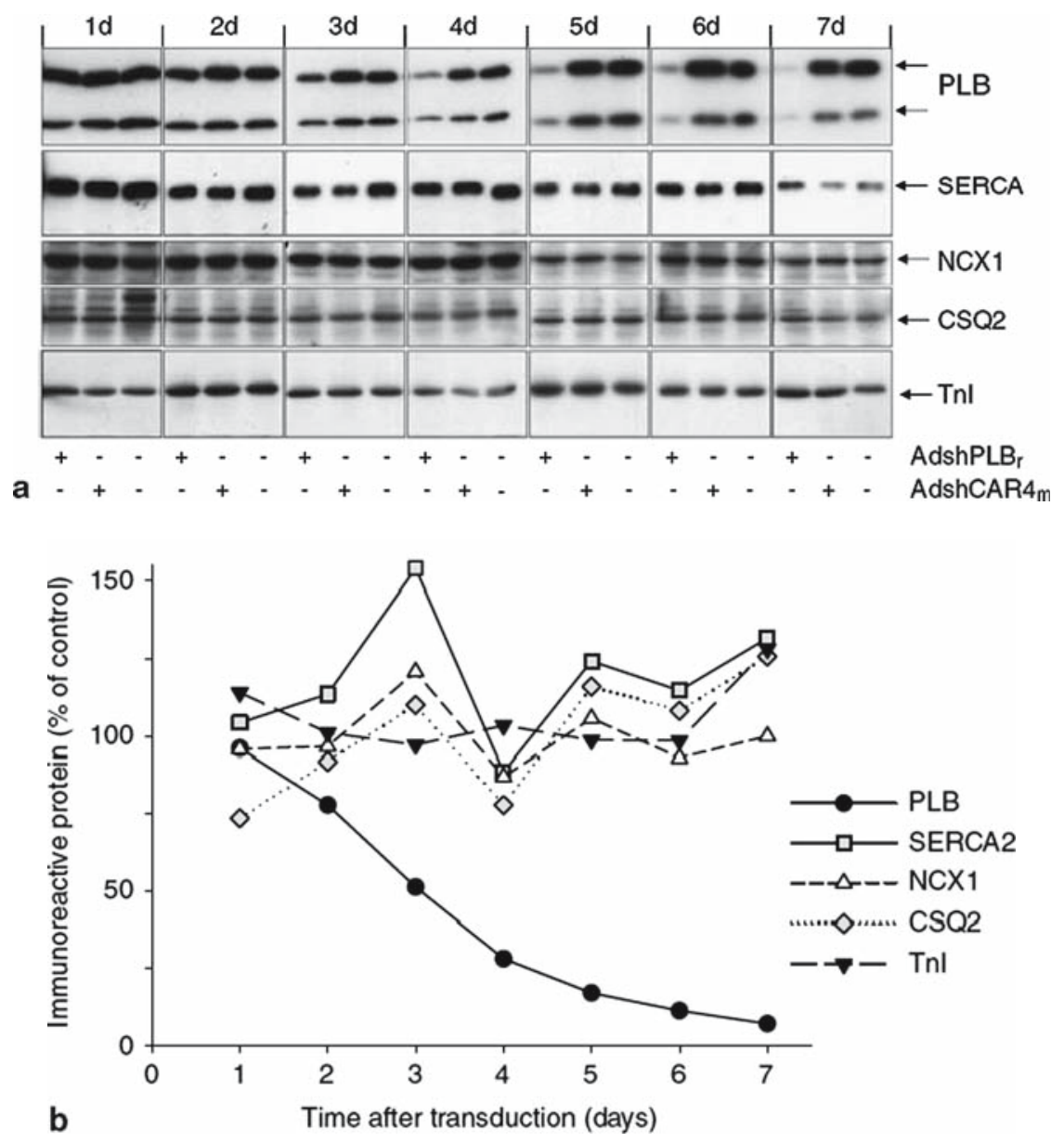

Fig. 12 Time-dependency of AdshPLBr-mediated PLB protein downregulation. (a) Western blot analysis of PLB protein expression. PNCMs were transduced with AdshPLBr or a control vector at an MOI of 100. Cells were harvested at indicated time points after transduction and Westernblots carried out. Significant PLB downregulation became visible 3 days post-transduction while the expression levels of SERCA, NCX1, TnI, and CSQ2 were unaffected during the 7-day investigation period (b) Relative expression levels of PLB, SERCA, NCX1, TnI, and CSQ2 protein normalized to the respective Western blot signals at the corresponding time points in control vector-transduced PNCMs

As shown in Fig. 13b, a linear relationship between the relative rates of $\mathrm{Ca}^{2+}$ uptake and the respective relative PLB protein levels was observed in AdshPLBrtransduced PNCMs. In addition we investigated the degree of stimulation of $\mathrm{Ca}^{2+}$ uptake by protein kinase A (PKA)-dependent in vitro phosphorylation following transduction of PNCMs with AdshPLBr at an MOI of 100. On day 1 after transduction the PKA-induced increase in the rate of $\mathrm{Ca}^{2+}$ uptake was $82 \%$ both in 

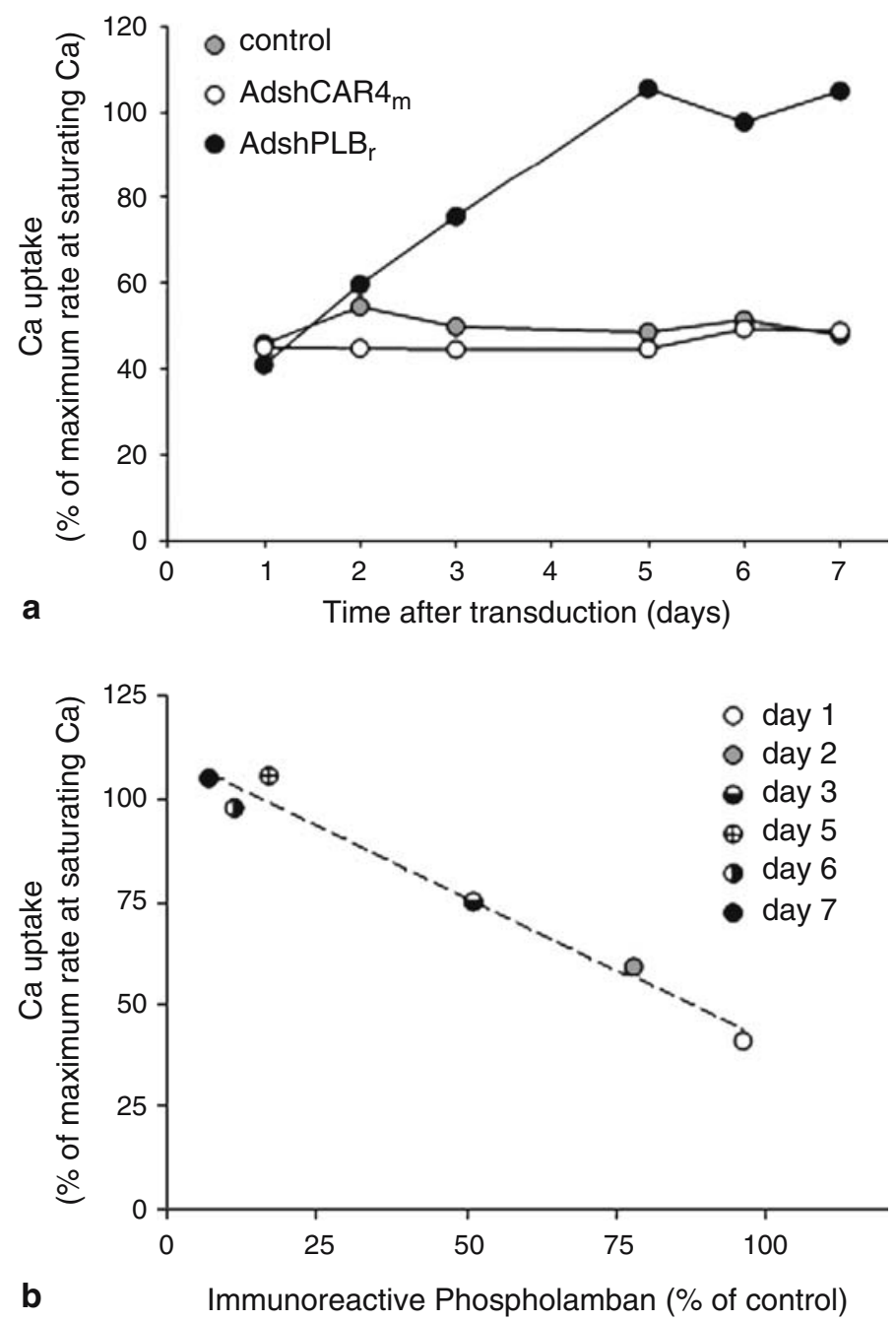

Fig. $13 \mathrm{SR}_{\mathrm{Ca}^{2+}}$ uptake in PNCM homogenates after AdshPLBr-mediated PLB silencing. (a) Relative $\mathrm{Ca}^{2+}$ uptake at $0.34 \mu \mathrm{M}$ free $\mathrm{Ca}^{2+}$ in homogenates of PNCMs. PNCMs were transduced with AdshPLBr or a control vector at an MOI of 100. Cells were harvested at indicated time points and $\mathrm{SR} \mathrm{Ca}^{2+}$ uptake rates were determined at submicromolar $(0.34 \mu \mathrm{M})$ and saturating $(3.68 \mu \mathrm{M})$ free $\mathrm{Ca}^{2+}$ concentrations. Values after $3 \mathrm{~min}$ of uptake were normalized to the maximum uptake at a saturating $\mathrm{Ca}^{2+}$ of $3.68 \mu \mathrm{M}$. Control Non-transduced cells. (b) Relative $\mathrm{Ca}^{2+}$ uptake versus percent change in PLB protein in PNCMs homogenates on days 1-7 post-transduction. Data were calculated from the results obtained from $\mathrm{Ca}^{2+}$ uptake experiments (a) and PLB protein expression (Fig. 12b)

non-transduced and in AdshPLBr-transduced PNCMs. In homogenates of AdshPLBrtransduced PNCMs this PKA stimulation became completely lost around day 4/5 after transduction, whereas approximately 1.5-fold stimulation of $\mathrm{Ca}^{2+}$ uptake was observed on days 4-7 in non-transduced PNCMs (Fig. 14). 


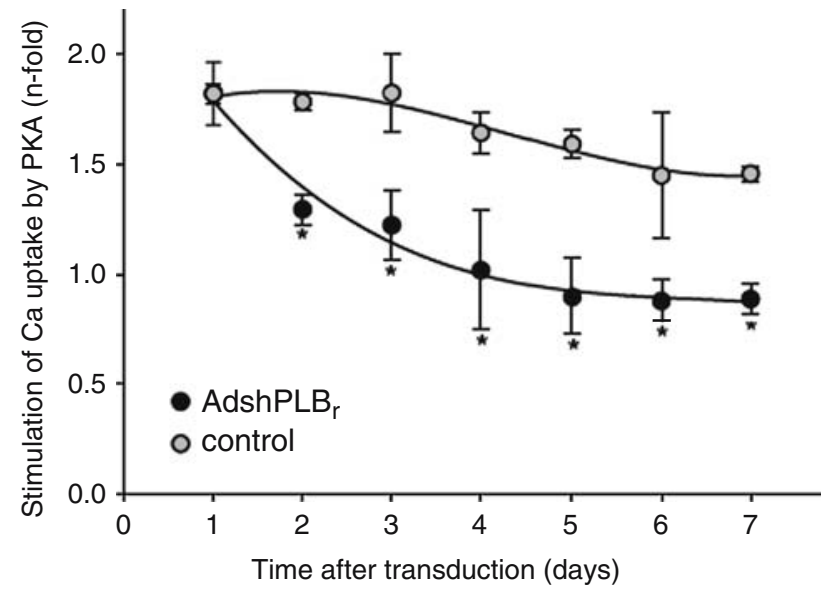

Fig. 14 Stimulation of $\mathrm{Ca} 2+$ uptake in PNCMs homogenates by the catalytic subunit of PKA. Values in the presence of added PKA normalized to those measured in the presence of $2 \mu \mathrm{M}$ synthetic PKA peptide inhibitor. Shown are means \pm SD for four separate transductions. Control Non-transduced cells

\subsection{Discussion of an RNAi-Based Approach to Heart Failure Therapy}

We demonstrated highly efficient and specific ablation of endogenous PLB expression in PNCMs by PLB-shRNA expressed from an AdV. shRNA transcription resulted in downregulation of endogenous PLB-mRNA below $0.1 \%$ of baseline, persisting for 13 days. No changes of the expression of other cardiac proteins including $\mathrm{Ca}^{2+}$ handling proteins occurred, indicating high target specificity of the PLB-shRNA vector. A control vector had no effect on PLB indicating absence of unspecific effects of shRNA per se on PNCMs. At the functional level, the SERCA2a $\mathrm{Ca}^{2+}$ affinity was markedly increased after PLB silencing accompanied by a loss of responsiveness to PKA-dependent stimulation of $\mathrm{Ca}^{2+}$ uptake. SERCA2a and PLB form a functional complex regulating $\mathrm{Ca}^{2+}$ uptake. Altered levels of either protein may therefore have profound effects by changing the PLB/ SERCA2a ratio and thereby altering intracardiac $\mathrm{Ca}^{2+}$ homeostasis. This was demonstrated in normal and diseased hearts by use of transgenic animal models or gene transfer approches targeting PLB or SERCA2a (Iwanaga et al. 2004; delMonte et al. 2001; Vetter et al. 2002; Miyamoto et al. 2000). Reduction of the PLB/SERCA2a ratio was achieved by classical overexpression of cDNAs encoding SERCA2a, a dominant negative PL mutant, or PLB-targeted antibodies. We and others have employed antisense RNAs directed against PLB-mRNA (Eizema et al. 2000; He et al. 1999).

In contrast, RNAi-based therapeutic strategies are not yet widely employed in the cardiovascular field. Synthetic siRNAs were recently employed to downregulate 
PLB in cardiomyocytes (Watanabe et al. 2004). Since siRNAs enable only transient gene silencing, we have developed an AdV generating shRNAs mediating efficient, specific, and stable PLB silencing. With respect to the duration of silencing a striking difference between previous work using synthetic siRNAs (Watanabe et al. 2004) and the current AdV-based approach was observed. siRNAs resulted in downregulation of PLB-mRNA to $5 \%$ of baseline within $12 \mathrm{~h}$, but this effect was almost completely lost after 4 days. In contrast, PLB-mRNA levels remained below $0.1 \%$ of baseline over a 2-week period after PLB-shRNA-AdV treatment. Vector-based shRNA generation was clearly superior to synthetic siRNAs with respect to silencing stability. With respect to the efficacy of silencing the PLB-shRNA-AdV resulted in $>99.9 \%$ ablation of PLB-mRNA, whereas a former study using a PLB-antisenseRNA-AdV achieved maximal PLB-mRNA ablation $\approx 75 \%$ (Eizema et al. 2000). In line with previous reports, we found PLB ablation to improve SERCA2a dependent intracellular $\mathrm{Ca}^{2+}$ handling. Significant upregulation of SERCA2a Ca ${ }^{2+}$ affinity was first observed when PLB protein was down to $50 \%$ of baseline. Interestingly, PLB downregulation to $20 \%$ was sufficient to mediate maximal increase in the $\mathrm{Ca}^{2+}$ affinity of SERCA2a, further reduction had no additional effect. There was a steady loss of responsiveness to PKA-dependent $\mathrm{Ca}^{2+}$ uptake stimulation by PLB-shRNA, and complete loss of responsiveness was observed on day 4-7. There was no loss of responsiveness to PKA-dependent $\mathrm{Ca}^{2+}$ uptake stimulation in the control groups. This indicates that the RNAi-mediated PLB silencing was linked to the anticipated loss of responsiveness of the $\mathrm{SR} \mathrm{Ca}^{2+}$ transport system to PKA-dependent phosphorylation normally mediated through PLB.

Although RNAi is a powerful method for gene silencing, its implementation for therapeutic purposes in humans requires that two technical problems are solved. First, instability of chemically synthesized siRNAs in plasma and cells requires their repetitive administration in vivo. The loss of initially efficient target gene silencing in cardiomyocytes within 3 days allows an estimate of the required siRNA application frequency for this specific target tissue. The problem may be overcome by viral vector systems producing shRNAs over long time periods in vivo. For intermediate stability, AdVshRNA systems as used here may suffice, and even provide advantages over long-term stable AAV vectors (Wang et al. 2005; Minamisawa et al. 1999a,b) if the RNAi effect is needed only temporarily in an acute and potentially reversible condition (e.g., heart failure due to viral or autoimmune myocarditis). Second, cardiac targeting of synthetic siRNAs is currently only possible by experimental methods unsuitable for possible transfer to any clinical setting (Lewis et al. 2002). In contrast, recent developments in vector technology (e.g., pseudotyped AAVs with cardiotropic properties) are likely to allow cardiac targeting of transgene and shRNA expression cassettes by simple intravenous injection (Inagaki et al. 2006; Pacak et al. 2006). Importantly, the small expression cassette and specific PLB-shRNA sequence used in this study can easily be incorporated in different AAV and other vectors selected for purpose. Within the framework of our proof-of-concept study which introduces a novel PL-shRNA tool we have not performed in vivo work, since the efficacy of PLB ablation for heart failure therapy in animal models has already been demonstrated (Iwanaga et al. 2004; delMonte et al. 2001; Vetter et al. 2002; Miyamoto et al. 2000). In future cardiac gene therapy studies, we will 
use pseudotyped AAV8 and AAV9 vectors, since two recent studies (Inagaki et al. 2006; Pacak et al. 2006) have demonstrated important advantages of these pseudotypes over lentiviruses, adenoviruses, and previously used AAV vectors. One remaining challenge is the modification of the cassette in such a way as to allow exogenously regulatable shRNA expression and adjustment of the degree of PLB modulation to changing physiological conditions. This appears to be particularly important in humans since chronic PLB deficiency due to genomic mutations was associated with cardiomyopathies (Schmitt et al. 2003; Zhao et al. 2006; Haghighi et al. 2003, 2006). Whereas in mice complete knockout of PLB (as may also be achieved by RNAi) was able to rescue the severe cardiomyopathic phenotype of MLP knockout mice (Minamisawa 1999a,b), suggesting that unregulated PLB silencing is appropriate in this species, application in humans most probably requires regulatable RNAi. Recent studies by our group have extended the repertoire of RNAi vector for the suppression of PLB by a series of AAV9based vectors. A long-term in vivo study of both the original AdV described above and analogous AAV9 vectors have demonstrate that over periods of 1 month (for the AdV vector) and 3 months (for the AAV9 vector) these RNAi vector significantly improve systolic and diastolic cardiac function, reduce cardiac hypertrophy and dilation, and improve survival (in the case of the AAV vectors only) (unpublished data). Furthermore, the catecholamine-induced deregulations of several functionally relevant miRNA in cardiomyocytes were restore to normal by RNAi vector treatment (unpublished data).

\section{Clinical Perspectives and Unanswered Questions}

\subsection{Three Levels of Cardiac Targeting}

During the past decade, the molecular and structural foundations and the key problems associated with cardiac gene therapy - and thereby also many of those encountered in regulatory RNA-based approaches - have been defined in considerable detail. It has become obvious that efficient and selective transport of any regulatory RNA or cDNA vector to the myocardium (Targeting) is a first key issue and major problem. Future clinical cardiac RNA and gene therapy protocols will probably involve direct intracoronary vector infusion to achieve target selectivity (level I of targeting). This may be enhanced by the use cardiac-specific promotors resulting in further confinement of regulatory RNA or cDNA expression (level II). Importantly, for regulatory RNA, e.g., shRNA transcription common tissue-specific type II polymerase promoters cannot be used since proper shRNA transcription has until recently only been achieved with type III promoters none of which was tissue-specific. This problem may be overcome, however, by the introduction of microRNA-based shRNA expression systems which also allow the use of cardiac-specific and drug-regulatable type II polymerase promotors. This makes level II of targeting also available for regulatory RNA-based therapies. Another major breakthrough in the field of cardiac RNA and gene therapy was the discovery that certain pseudotyped AAV vectors (in particular 
AAV9 and derivates thereof) are cardiotropic even after simple intravenous injection (level III of targeting). Although AAV9 is not yet absolutely cardiac-specific but also reaches the liver by the intravenous route, further modification of the AAV capsid may solve or alleviate this issue. Currently, the use of AAV9 in combination with level I and II targeting already enables highly cardiac-specific RNA and gene therapy protocols one of which is evaluated in a first clinical trial.

\subsection{Stability and Control of Regulatory RNA and Gene Therapy}

Depending on the target disease, the therapeutic goal may be achieved by regulatory RNA or transgene expression for only few days or weeks or require long-term of even life-long stability. Currently available adenovectors are capable of achieving the short-term goal which may also suffice in bridge-to-recovery or bridge-to-transplant situations. Nevertheless, even for short-term therapies the generally better safety profile of AAV vectors will lead to the use of this vector type in any clinical RNA or gene therapy protocol. For all long-term therapies AAV vectors are a priori the system of choice due to their impressive long-term cardiac stability and function in several experimental studies. Whereas control of transgene expression is available for some years via drug-regulatable system, this goal has only recently been achieved for regulatory RNA therapies. As for conventional gene therapy, optimized clinical RNA therapy protocols are also likely to involve drug-regulatable systems since under certain conditions shRNAs (and likely also other regulatory RNA, e.g., miRNAs) have shown serious adverse effects. The option to shut off their expression will therefore significantly add to therapeutic safety in the clinical setting.

\subsection{Practical Issues of Clinical Translation}

Even when the above basic issues are solved satisfactorily, the intensity of efforts to establish a regulatory RNA or gene therapeutic regime in the clinic will be critically influenced by four determinants. First, that the specific therapeutic target is not accessible by other means, e.g., conventional pharmacotherapy. Second, that the target disease is sufficiently severe to justify the efforts and risks of any experimental therapeutic approach. Third, that the number of patients which may possibly benefit from the experimental therapy to be established is relatively high since this will greatly facilitate fundraising and incorporation of industrial partners. Fourth, any therapeutic protocol which addresses a pathomechanism (e.g., disturbed cardiac $\mathrm{Ca}^{2+}$ homeostasis) common to cardiac diseases of diverse etiologies will have a broader range of application than strategies which target specific pathogenic steps, and need to be tailor-made for every different type of pathogenesis. Without doubt, in cardiovascular medicine in general and specifically in the fields of cardio-myopathies and heart failure, a large number of patients are in urgent need of improved treatment, and the first regulatory RNA and 
gene therapeutic protocols adequately fulfill the above criteria. Thus, actual clinical translation of first therapies of this type is likely to occur within the next decade.

\section{References}

Afione S, Conrad C et al (1996) In vivo model of adeno-associated virus vector persistence and rescue. J Virol 70(5):3235-3241

Anderson J, Akkina R (2005) HIV-1 resistance conferred by siRNA cosuppression of CXCR4 and CCR5 coreceptors by a bispecific lentiviral vector. AIDS Res Ther 2(1):1

Arrighi JF, Pion M et al (2004) Lentivirus-mediated RNA interference of DC-SIGN expression inhibits human immunodeficiency virus transmission from dendritic cells to T cells. J Virol 78(20): $10848-10855$

Auricchio A, Acton P et al (2003) In vivo quantitative noninvasiive imaging of gene transfer by single-photon emission computerized tomography. Human Gene Ther 14:255-261

Beeri R, Guerrero L et al (2002) New efficient catheter-based system for myoccardial gene delivery. Circulation 106:1756-1759

Bergelson J, Cunningham J et al (1997) Isolation of a common receptor for coxsackie B viruses and adenoviruses 2 and 5. Science 275:1320-1323

Bergelson J, Krithivas A et al (1998) The murine CAR homolog is a receptor for coxsackie B viruses and adenoviruses. J Virol 72(1):415-419

Bitko V, Musiyenko A et al (2005) Inhibition of respiratory viruses by nasally administered siRNA. Nat Med 11(1):50-55

Bliznakov E (2002) Therapeutic angiogenesis: hope or hype. Circulation 106(25):e220-e221

Boden D, Pusch O et al (2003) Human immunodeficiency virus type 1 escape from RNA interference. J Virol 77(21):11531-11535

Boden D, Pusch O et al (2007) Overcoming HIV-1 resistance to RNA interference. Front Biosci 12:3104-3116

Boekstegers P, vonDegenfeld G et al (2000) Myocardial gene transfer by selective pressure-regulated retroinfusion of coronary veins. Gene Ther 7:232-240

Bowles NE, Richardson PJ et al (1986) Detection of coxsackie-B-virus-specific RNA sequences in myocardial biopsy samples from patients with myocarditis and dilated cardiomyopathy. Lancet 1(8490):1120-1123

Bowles NE, Ni J et al (2003) Detection of viruses in myocardial tissues by polymerase chain reaction. evidence of adenovirus as a common cause of myocarditis in children and adults. J Am Coll Cardiol 42(3):466-472

Büning H, Ried M et al (2003) Receptor targeting of adeno-associated virus vectors. Gene Ther 10:1142-1151

Carmona S, Ely A et al (2006) Effective inhibition of HBV replication in vivo by anti-HBx short hairpin RNAs. Mol Ther 13(2):411-421

Check E (2003) Cancer risk prompts US to curb gene therapy. Nature 422:7

Chen JW, Zhou B et al (2006) Cardiomyocyte-specific deletion of the coxsackievirus and adenovirus receptor results in hyperplasia of the embryonic left ventricle and abnormalities of sinuatrial valves. Circ Res 98:923-930

Chen CC, Ko TM et al (2007) Long-term inhibition of hepatitis B virus in transgenic mice by doublestranded adeno-associated virus 8-delivered short hairpin RNA. Gene Ther 14(1):11-19

Chtarto A, Bender $\mathrm{H}$ et al (2003) Tetracycline-inducible transgene expression mediated by a single AAV vector. Gene Ther 10:84-94

Cohen C, Shieh J et al (2001) The coxsackievirus and adenovirus receptor is a transmembrane component of the tight junction. Proceedings of the National Academy of Science USA 98(26):15191-15196

Communal C, Huq F et al (2003) Decreased efficiency of adenovirus-mediated gene transfer in aging cardiomyocytes. Circulation 107(8):1170-1175 
Coyne CB, Bergelson JM (2006) Virus-induced Abl and Fyn kinase signals permit coxsackievirus entry through epithelial tight junctions. Cell 124(1):119-131

Curiel D (1999) Considerations and challenges for the achievement of targeted gene delivery (Perspective) Gene Ther 6(9):1497-1498

Davidson M, Jones J et al (2001) Cardiac gene delivery with cardiopulmonary bypass. Circulation 104:131-133

Dechecchi MC, Melotti P et al (2001) Heparan sulfate glycosaminoglycans are receptors sufficient to mediate the initial binding of adenovirus types 2 and 5. J Virol 75(18):8772-8780

delMonte F, Williams E et al (2001) Improvement in survival and cardiac metabolism after gene transfer of sarcoplasmic reticulum $\mathrm{Ca}^{2+}$ ATPase in a rat model of heart failure. Circulation 104:1424-1429

delMonte F, Harding S et al (2002) Targeting phospholamban by gene transfer in human heart failure. Circulation 105:904-907

Dieterle T, Meyer M et al (2005) Gene transfer of a phospholamban-targeted antibody improves calcium handling and cardiac function in heart failure. Cardiovasc Res 67(4):678-688

Donahue J, Kikkawa K et al (1997) Ultrarapid, highly efficient viral gene transfer to the heart. Proc Natl Acad Sci USA 94:4664-4668

Dorner AA, Wegmann F et al (2005) Coxsackievirus-adenovirus receptor (CAR) is essential for early embryonic cardiac development. J Cell Sci 118(Pt 15):3509-3521

Eizema K, Fechner H et al (2000) Adenovirus-based phospholamban-antisense-mRNA expression as a novel approach to improve cardiac contractile dysfunction - comparison of a constitutive viral versus an endothelin-1-responsive cardiac promoter. Circulation 101:2193-2199

Fechner H, Haack A et al (1999) Expression of coxsackie-adenovirus-receptor and $\alpha$-integrin does not correlate with adenovector targeting in vivo indicating anatomical vector barriers. Gene Ther 6(9):1520-1535

Fechner H, Noutsias M et al (2003a) Induction of coxsackievirus-adenovirus-receptor expression during myocardial tissue formation and remodeling: identification of a cell-to-cell contactdependent regulatory mechanism. Circulation 107(6):876-882

Fechner H, Wang X et al (2003b) A novel tetracycline-controlled transactivator-transrepressor system enables external control of oncolytic adenovirus replication. Gene Ther 10:1680-1690

Fechner H, Suckau L et al (2007) Highly efficient and specific modulation of cardiac calcium homeostasis by adenovector-derived short hairpin RNA targeting phospholamban. Gene Ther 14:211-218

Franz W, Rothmann T et al (1997) Analysis of tissue-specific gene delivery by recombinant adenoviruses containing cardiac-specific promoters. Cardiovascular Research 35:560-566

Gao X, Wang H et al (2004) Inhibition of Epstein-Barr virus (EBV) reactivation by short interfering RNAs targeting p38 mitogen-activated protein kinase or c-myc in EBV-positive epithelial cells. J Virol 78(21):11798-11806

Giordano F, Ping P et al (1996) Intracoronary gene transfer of fibroblast growth factor-5 increases blood flow and contractile function in an ischemic region of the heart. Nature Medicine 2(5):534-539

Gitlin L, Stone JK et al (2005) Poliovirus escape from RNA interference: short interfering RNAtarget recognition and implications for therapeutic approaches. J Virol 79(2):1027-1035

Grines C, Watkins M et al (2002) Angiogenic gene therapy (AGENT) trial in patients with stable angina pectoris. Circulation 105:1291-1297

Hacein-Bey-Abina S, LeDeist F et al (2002) Sustained correction of X-linked severe combined immunodeficiency by ex vivo gene therapy. N Engl J Med 346(16):1185-1193

Hacein-Bey-Abina S, vonKalle C et al (2003) A serious adverse event after successful gene therapy for X-linked severe combined immunodeficiency. N Engl J Med 348(3):255-256

Haghighi K, Kolokathis F et al (2003) Human phospholamban null results in lethal dilated cardiomyopathy revealing a critical difference between mouse and human. J Clin Invest 111(6):869-876

Haghighi K, Kolokathis F et al (2006) A mutation in the human phospholamban gene, deleting arginine 14, results in lethal, hereditary cardiomyopathy. Proc Natl Acad Sci USA 103(5):1388-1393

Hajjar R, Schmidt U et al (1998) Modulation of ventricular function through gene transfer in vivo. Proc Natl Acad Sci USA 95:5251-5256 
Hayafune M, Miyano-Kurosaki N et al (2006a) HIV gene therapy using RNA virus systems. Nucleic Acids Symp Ser (Oxf) (50):79-80

Hayafune M, Miyano-Kurosaki N et al (2006b) Silencing of HIV-1 gene expression by two types of siRNA expression systems. Antivir Chem Chemother 17(5):241-249

$\mathrm{He} \mathrm{H}$, Meyer M et al (1999) Effects of mutant and antisense RNA of phospholamban on SR $\mathrm{Ca}^{2+}-$ ATPase activity and cardiac myocyte contractility. Circulation 100:974-980

Hedman M, Hartikainen J et al (2003) Safety and feasibility of catheter-based local intracoronary vascular endothelial growth factor gene transfer in the prevention of postangioplasty and instent restenosis and in the treatment of chronic myocardial ischemia - phase II results of the Kuopio Angiogenesis Trial (KAT). Circulation 107:2677-2683

Henderson S, Spencer M et al (1989) Structure, organization, and expression of the rat cardiac myosin light chain-2 gene. Identification of a 250-base pair fragment which confers cardiacspecific expression. J Biol Chem 264(30):18142-18148

Herzog R, Fields P et al (2002) Influence of vector dose on factor IX-specific T and B cell responses in muscle-directed gene therapy. Hum Gene Ther 13:1281-1291

Honda T, Saitoh $\mathrm{H}$ et al (2000) The coxsackievirus-adenovirus receptor protein as a cell adhesion molecule in the developing mouse brain. Brain Res Mol Brain Res 77:19-28

Hong SS, Karayan L et al (1997) Adenovirus type 5 fiber knob binds to MHC class I alpha2 domain at the surface of human epithelial and B lymphoblastoid cells. EMBO J 16(9):2294-2306

Hoshijima M, Ikeda Y et al (2002) Chronic suppression of heart-failure progression by a pseudophosphorylated mutant of phospholamban via in vivo cardiac rAAV gene delivery. Nat Med 8(8):864-871

Ikeda Y, Gu Y et al (2002) Restoration of deficient membrane proteins in the cardiomyopathic hamster by in vivo cardiac gene transfer. Circulation 105:502-508

Inagaki K, Fuess S et al (2006) Robust systemic transduction with AAV9 vectors in mice: efficient global cardiac gene transfer superior to that of AAV8. Mol Ther 14(1):45-53

Inubushi M, Wu J et al (2003) Positron-emission tomography reporter gene expression imaging in rat myocardium. Circulation 107:326-332

Iwanaga Y, Hoshijima M et al (2004) Chronic phospholamban inhibition prevents progressive cardiac dysfunction and pathological remodeling after infarction in rats. J Clin Invest 113(5):727-736

Kameoka M, Nukuzuma S et al (2004) RNA interference directed against Poly(ADP-Ribose) polymerase 1 efficiently suppresses human immunodeficiency virus type 1 replication in human cells. J Virol 78(16):8931-8934

Kandolf R (2004) [Virus etiology of inflammatory cardiomyopathy]. Dtsch Med Wochenschr 129(41):2187-2192

Kawada T, Nakazawa M et al (2002) Rescue of hereditary form of dilated cardiomyopathy by rAAV-mediated somatic gene therapy: Amelioration of morphological findings, sarcolemmal permeability, cardiac performances, and the prognosis of TO-2 hamsters. Proc Natl Acad Sci USA 99:901-906

Kay M, Manno C et al (2000) Evidence for gene transfer and expression of factor IX in haemophilia B patients treated with an AAV vector. Nat Genet 24:257-261

Koch W, Lefkowitz R et al (2000) Functional consequences of altering myocardial adrenergic receptor signaling. Annu Rev Physiol 62:237-260

Kohn D, Sadelain M et al (2003) Occurrence of leukaemia following gene therapy of X-linked SCID. Nat Rev Cancer 3:477-488

Kühl U, Pauschinger M et al (2003) Interferon- $\beta$ treatment eliminates cardiotropic viruses and improves left ventricular function in patients with myocardial persistence of viral genomes and left ventricular dysfunction. Circulation 107(22):2793-2798

Kühl U, Pauschinger M et al (2005a) High prevalence of viral genomes and multiple viral infections in the myocardium of adults with idiopathic left ventricular dysfunction. Circulation 111:887-893

Kühl U, Pauschinger M et al (2005b) Virus persistence in the myocardium is associated with progressive cardiac dysfunction. Circulation 112(13):1965-1970

Kypson A, Hendrickson S et al (1999) Adenovirus-mediated gene transfer of the b2-adrenergic receptor to donor hearts enhances cardiac function. Gene Ther 6:1298-1304

Lehrman S (1999) Virus treatment questioned after gene therapy death. Nature 401:517-518 
Leung RK, Whittaker PA (2005) RNA interference: from gene silencing to gene-specific therapeutics. Pharmacol Ther 107(2):222-239

Lewis D, Hagstrom J et al (2002) Efficient delivery of siRNA for inhibition of gene expression in postnatal mice. Nat Genet 32:107-108

Li E, SL Brown et al (2001) Integrin alpha(v)beta1 is an adenovirus coreceptor. J Virol 75(11):5405-5409

$\mathrm{Li} \mathrm{J}, \mathrm{Hu} \mathrm{SJ}$ et al (2005) Construction of phospholamban antisense RNA recombinant adeno-associated virus vector and its effects in rat cardiomyocytes. Acta Pharmacol Sin 26(1):51-55

Lu A, Zhang $\mathrm{H}$ et al (2004) Attenuation of SARS coronavirus by a short hairpin RNA expression plasmid targeting RNA-dependent RNA polymerase. Virology 324(1):84-89

Luo W, Grupp IL et al (1994) Targeted ablation of the phospholamban gene is associated with markedly enhanced myocardial contractility and loss of beta-agonist stimulation. Circ Res 75(3):401-409

Marshall E (2000) Gene therapy on trial. Science 288:951-957

Maurice J, Hata J et al (1999) Enhancement of cardiac function after adenoviral-mediated in vivo intracoronary $\beta 2$-adrenergic receptor gene delivery. J Clin Invest 104:21-29

McCaffrey AP, Nakai H et al (2003) Inhibition of hepatitis B virus in mice by RNA interference. Nat Biotechnol 21(6):639-644

Merl S, Michaelis C et al (2005) Targeting 2A protease by RNA interference attenuates coxsackieviral cytopathogenicity and promotes survival in highly susceptible mice. Circulation 111(13):1583-1592

Meyer M, Belke DD et al (2004) A recombinant antibody increases cardiac contractility by mimicking phospholamban phosphorylation. FASEB J 18(11):1312-1314

Miller D, Rutledge E et al (2002) Chromosomal effects of adeno-associated virus vector integration. Nat Genet 30:147-148

Miller DG, Petek LM et al (2004) Adeno-associated virus vectors integrate at chromosome breakage sites. Nat Genet 36(7):767-773

Minamisawa S, Hoshijima M et al (1999a) Genetic complementation identifies chronic phospholamban-sarcoplasmic reticulum calcium ATPas interaction as a critical calcium cycling defect in the progression of dilated cardiomyopathy. Cell 99(3):313-322

Minamisawa S, Hoshijima M et al (1999b) Chronic phospholamban-sarcoplasmic reticulum calcium ATPase interaction is the critical calcium cycling defect in dilated cardiomyopathy. Cell 99(3):313-322

Miyamoto M, delMonte F et al (2000) Adenoviral gene transfer of SERCA2a improves left-ventricular function in aortic-banded rats in transition to heart failure. Proc Natl Acad Sci USA 97:793-798

Monahan P, Samulski R et al (1998) Direct intramuscular injection with recombinant AAV vectors results in sustained expression in a dog model of hemophilia. Gene Ther 5(1):40-49

Murray JL, Mavrakis M et al (2005) Rab9 GTPase is required for replication of human immunodeficiency virus type 1, filoviruses, and measles virus. J Virol 79(18):11742-11751

Nakai H, Iwaki Y et al (1999) Isolation of recombinant adeno-associated virus vector-cellular DNA junctions from mouse liver. J Virol 73(7):5438-5447

Nakai H, Yant S et al (2001) Extrachromosomal recombinant adeno-associated virus vector genomes are primarily responsible for stable liver transduction in vivo. J Virol 75(15):6969-6976

Nakai H, Storm T et al (2003) Pathways of removal of free DNA vector ends in normal and DNA-PKcsdeficient SCID mouse hepatocytes transduced with rAAV vectors. Hum Gene Ther 14:871-881

Nicklin S, Baker A (2002) Tropism-modified adenoviral and adeno-associated viral vectors for gene therapy. Curr Gene Ther 2(3):273-293

Noutsias M, Fechner $\mathrm{H}$ et al (2001) Human coxsackie-adenovirus-receptor is co-localized with integrins $\alpha_{v} \beta_{3}$ and $\alpha_{v} \beta_{5}$ on the cardiomyocyte sarcolemma and upregulated in dilated cardiomyopathy - implications for cardiotropic viral infections. Circulation 104:275-280

Ogorelkova M, Zwaagstra J et al (2006) Adenovirus-delivered antisense RNA and shRNA exhibit different silencing efficiencies for the endogenous transforming growth factor-beta (TGF-beta) type II receptor. Oligonucleotides 16(1):2-14

Pacak CA, Mah CS et al (2006) Recombinant adeno-associated virus serotype 9 leads to preferential cardiac transduction in vivo. Circ Res 99(4):e3-e9 
Park WS, Hayafune M et al (2003) Specific HIV-1 env gene silencing by small interfering RNAs in human peripheral blood mononuclear cells. Gene Ther 10(24):2046-2050

Perabo L, Büning $\mathrm{H}$ et al (2003) In vitro selection of viral vectors with modified tropism: the adeno-associated virus display. Mol Ther 8(1):151-157

Piacentino V 3rd, Weber CR et al (2003) Cellular basis of abnormal calcium transients of failing human ventricular myocytes. Circ Res 92(6):651-658

Ping YH, Chu CY et al (2004) Modulating HIV-1 replication by RNA interference directed against human transcription elongation factor SPT5. Retrovirology 1(1):46

Poller W, Fechner H et al (2002a) The molecular basis of cardiotropic viral infections. Eur Heart J 4(Suppl I):I18-I30

Poller W, Fechner H et al (2002b) Highly variable expression of virus receptors in the human cardiovascular system. Implications for cardiotropic viral infections and gene therapy. Z Kardiol 91(12):978-991

Ponnazhagan S, Hoover F (2004) Delivery of DNA to tumor cells in vivo using adeno-associated virus. Methods Mol Biol 246:237-243

Ponnazhagan S, Mahendra G et al (2002) Conjugate-based targeting of recombinant adeno-associated virus type 2 vectors by using avidin-linked ligands. J Virol 76(24):12900-12907

Price R, Skyba D et al (1998) Delivery of colloidal particles and red blood cells to tissue through microvessel ruptures created by targeted microbbubble destruction with ultrasound. Circulation 98:1264-1267

Rabinowitz J, Rolling F et al (2002) Cross-packaging of a single adeno-associated virus (AAV) type 2 vector genome into multiple AAV serotypes enables transduction with broad specificity. J Virol 76(2):791-801

Raper S, Yudkoff M et al (2002) A pilot study of in vivo liver-directed gene transfer with an adenoviral vector in partial ornithine transcarbamylase deficiency. Hum Gene Ther 13:163-175

Reid T, Warren R et al (2002) Intravascular adenoviral agents in cancer patients: lessons from clinical trials. Cancer Gene Ther 9(12):979-986

Reynolds P, Nicklin S et al (2001) Combined transductional and transcriptional targeting. Nat Biotechnol 19:838-842

Reynolds A, Leake D et al (2004) Rational siRNA design for RNA interference. Nat Biotechnol 22(3):326-330

Rockman H, Chien K et al (1998) Expression of a $\beta$-adrenergic receptor kinase 1 inhibitor prevents the development of myocardial failure in gene-targeted mice. Proc Natl Acad Sci USA 95:7000-7005

Rutledge E, Russell D (1997) Adeno-associated virus vector integration junctions. J Virol 71(11):8429-8436

Rutledge EA, Halbert CL et al (1998) Infectious clones and vectors derived from adeno-associated virus (AAV) serotypes other than AAV type 2. J Virol 72(1):309-319

Sabariegos R, Gimenez-Barcons M et al (2006) Sequence homology required by human immunodeficiency virus type 1 to escape from short interfering RNAs. J Virol 80(2):571-577

Schmidt U, Hajjar RJ et al (1999) Human heart failure: cAMP stimulation of SR Ca ${ }^{2+}$-ATPase activity and phosphorylation level of phospholamban. Am J Physiol 277(2 Pt 2):H474-H480

Schmitt J, Kamisago M et al (2003) Dilated cardiomyopathy and heart failure caused by a mutation in phospholamban. Science 299:1410-1413

Shaw CA, Holland PC et al (2004) Isoform-specific expression of the coxsackie and adenovirus receptor (CAR) in neuromuscular junction and cardiac intercalated discs. BMC Cell Biol 5(1):42

Shi W, Bartlett J (2003) RGD inclusion in VP3 provides adeno-associated virus type 2 (AAV2)-based vectors with a heparan sulfate-independent cell entry mechanism. Mol Ther 7(4):515-525

Simons M, Bonow R et al (2000) Clinical trials in coronary angiogenesis: Issues, problems, consensus. An expert panel summary. Circulation 102:e73-e86

Sollerbrant K, Raschperger E et al (2003) The coxsackievirus and adenovirus receptor (CAR) forms a complex with the PDZ domain-containing protein ligand-of-numb protein-X (LNX) J Biol Chem 278(9):7439-7444

Srour M, Fechner H et al (2003) Regulation of human factor IX expression using a doxycyclineinducible gene expression system. Thromb Haemost 90:398-405 
Sun J, Chatterjee $S$ et al (2002) Immunogenic issues concerning recombinant adeno-associated virus vectors for gene therapy. Curr Gene Ther 2:485-500

Sun J, Anand-Jawa V et al (2003) Immune responses to adeno-associated virus and its recombinant vectors. Gene Ther 10:964-976

Takahashi H, Hirai Y et al (2002) Long-term systemic therapy of Fabry disease in a knockout mouse by adeno-associated virus-mediated muscle-directed gene transfer. Proc Natl Acad Sci USA 99(21):13777-13782

Takigawa Y, Nagano-Fujii M et al (2004) Suppression of hepatitis C virus replicon by RNA interference directed against the NS3 and NS5B regions of the viral genome. Microbiol Immunol 48(8):591-598

Tomko R, Xu R et al (1997) HCAR and MCAR: the human and mouse cellular receptors for subgroup $\mathrm{C}$ adenoviruses and group B coxsackieviruses. Proc Natl Acad Sci USA 94:3352-3356

Vale P, Losordo D et al (1999) Catheter-based myocardial gene transfer using nonfluoroscopic electromechanical left ventricular mapping. J Am Coll Cardiol 34(1):246-254

Vetter R, Rehfeld U et al (2002) Transgenic overexpression of the sarcoplasmic reticulum $\mathrm{Ca}^{2+}$ ATPase improves reticular $\mathrm{Ca}^{2+}$ handling in normal and diabetic rat hearts. FASEB J 16:1657-1659

Wang Z, Zhu T et al (2005) Adeno-associated virus serotype 8 efficiently delivers genes to muscle and heart. Nat Biotechnol 23(3):321-328

Watanabe A, Arai M et al (2004) Phospholamban ablation by RNA interference increases Ca2+ uptake into rat cardiac myocyte sarcoplasmic reticulum. J Mol Cell Cardiol 37(3):691-698

Weinberg MS, Ely A et al (2007) Specific inhibition of HBV replication in vitro and in vivo with expressed long hairpin RNA. Mol Ther 15(3):534-541

Werk D, Schubert S et al (2005) Developing an effective RNA interference strategy against a plusstrand RNA virus: silencing of coxsackievirus B3 and its cognate coxsackievirus-adenovirus receptor. Biol Chem 386(9):857-863

White D, Hata J et al (2000) Preservation of myocardial b-adrenergic receptor signaling delays the development of heart failure after myocardial infarction. Proc Natl Acad Sci USA 97(10):5428-5433

Wickham T (2000) Targeting adenovirus. Gene Ther 7:110-114

Wickham T, Mathias P et al (1993) Integrins $\alpha_{\mathrm{v}} \beta_{3}$ and $\alpha_{\mathrm{v}} \beta_{5}$ promote adenovirus internalization but not virus attachment. Cell 73(2):309-319

Wickham T, Tzeng E et al (1997) Increased in vitro and in vivo gene transfer by adenovirus vectors containing chimeric fiber proteins. J Virol 71:8221-8229

Wiebusch L, Truss M et al (2004) Inhibition of human cytomegalovirus replication by small interfering RNAs. J Gen Virol 85(Pt 1):179-184

Wilson JA, Richardson CD (2005) Hepatitis C virus replicons escape RNA interference induced by a short interfering RNA directed against the NS5b coding region. J Virol 79(11):7050-7058

Wolska BM, Arteaga GM et al (2002) Expression of slow skeletal troponin I in hearts of phospholamban knockout mice alters the relaxant effect of beta-adrenergic stimulation. Circ Res 90(8):882-888

Wu J, Inubushi M et al (2002) Positron emission tomography imaging of cardiac reporter gene expression in living rats. Circulation 106:180-183

Ylä-Herttuala S, Alitalo K (2003) Gene transfer as a tool to induce therapeutic vascular growth. Nat Med 9(6):694-701

Yoon JS, Kim SH et al (2004) Inhibition of herpesvirus-6B RNA replication by short interference RNAs. J Biochem Mol Biol 37(3):383-385

Yuan J, Cheung P et al (2005) Inhibition of coxsackievirus B3 replication by small interfering RNAs requires perfect sequence match in the central region of the viral positive strand. J Virol 79(4):2151-2159

Zen K, Liu Y et al (2005) Neutrophil migration across tight junctions is mediated by adhesive interactions between epithelial coxsackie and adenovirus receptor and a junctional adhesion molecule-like protein on neutrophils. Mol Biol Cell 16(6):2694-2703

Zhao W, Yuan Q et al (2006) The presence of Lys27 instead of Asn27 in human phospholamban promotes sarcoplasmic reticulum $\mathrm{Ca}^{2+}$-ATPase superinhibition and cardiac remodeling. Circulation 113(7):995-1004 